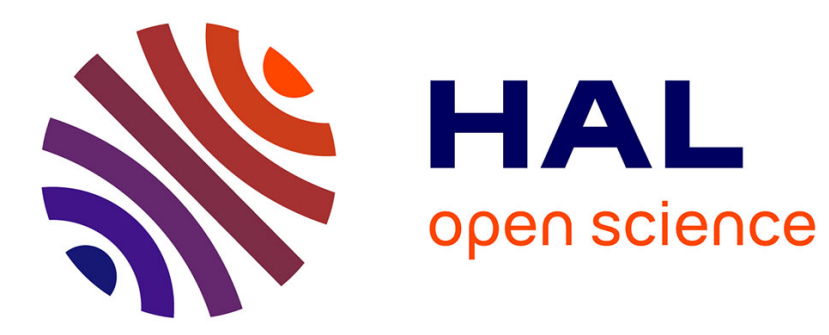

\title{
Constructive solution of a bilinear optimal control problem for a Schrödinger equation
}

\author{
Lucie Baudouin, Julien Salomon
}

\section{To cite this version:}

Lucie Baudouin, Julien Salomon. Constructive solution of a bilinear optimal control problem for a Schrödinger equation. Systems and Control Letters, 2008, 57 (6), pp. 453-464; 150. hal-00271940

\author{
HAL Id: hal-00271940 \\ https://hal.science/hal-00271940
}

Submitted on 10 Apr 2008

HAL is a multi-disciplinary open access archive for the deposit and dissemination of scientific research documents, whether they are published or not. The documents may come from teaching and research institutions in France or abroad, or from public or private research centers.
L'archive ouverte pluridisciplinaire HAL, est destinée au dépôt et à la diffusion de documents scientifiques de niveau recherche, publiés ou non, émanant des établissements d'enseignement et de recherche français ou étrangers, des laboratoires publics ou privés. 


\title{
Constructive solution of a bilinear optimal control problem for a Schrödinger equation
}

\author{
Lucie Baudouin* \\ LAAS - CNRS; Université de Toulouse; 7, avenue du Colonel Roche, F-31077 Toulouse, France. \\ Julien Salomon ${ }^{\dagger}$ \\ CEREMADE, Université Paris-Dauphine, Pl. du Mal Lattre de Tassigny, F-75775 Paris, France.
}

\begin{abstract}
Often considered in numerical simulations related to the control of quantum systems, the so-called monotonic schemes have not been so far much studied from the functional analysis point of view. Yet, these procedures provide an efficient constructive method for solving a certain class of optimal control problems. This paper aims both at extending the results already available about these algorithms in the finite dimensional case (i.e., the time-discretized case) and at completing those of the continuous case. This paper starts with some results about the regularity of a functional related to a wide class of model in quantum chemistry. Those enable us to extend an inequality due to Eojasiewicz to the infinite dimensional case. Finally, some inequalities proving the Cauchy character of the monotonic sequence are obtained, followed by an estimation of the rate of convergence.
\end{abstract}

Keywords: Bilinear optimal control, Łojasiewicz inequality, Monotonic schemes, Quantum systems, Schrödinger equation.

AMS Classification: 49M30, 49K20.

\section{Introduction}

Following the increasing interest of the chemists community for optimal control of quantum systems $[21,27]$ and the successful laboratory demonstration of control over molecular phenomena (see, e.g., $[1,7,32]$ and more recently $[12,31]$ ), some mathematical studies of the models involved in this topic have been carried out, see e.g. $[6,16]$. In this way, it has been proved in recent papers $[4,9]$ that a wide class of optimization problems considered by chemists are well posed. Yet, these proofs are not constructive and consequently do not give rise to concrete numerical methods to approximate their solutions.

On the other hand, at numerical simulation level [8, 22], various kind of procedures exist and show a good efficiency. Among them, the so-called monotonic algorithms have demonstrated their efficiency on several problems. In a recent paper, a study of the timediscretized algorithms [24] have been presented and first functional analysis results have been obtained about the continuous case [14, 25].

The aim of this paper is to complete these works by providing general proofs of convergence of the optimizing sequences. Consequently, we obtain a constructive method, independent of time or space discretization to compute critical points (and sometimes extrema, see

*e-mail: baudouin@laas.fr

†e-mail: julien.salomon@dauphine.fr 
Remark 3) of the cost functional under consideration.

Let us briefly present the monotonic schemes in the simple case of ordinary differential equations (ODE). Let $A, B, C$ be three square matrices in $\mathcal{M}_{n}(\mathbb{R}), C$ being symmetric positive, $\alpha>0$ and $T>0$. Consider the optimal control problem corresponding to the maximization of the functional $J$ defined by:

$$
J(v)=y(T) \cdot C y(T)-\alpha \int_{0}^{T} v^{2}(t) d t,
$$

where "." denotes the usual scalar product of $\mathbb{R}^{n}$. Here, the state $y:[0, T] \rightarrow \mathbb{R}^{n}$ and the control $v:[0, T] \rightarrow \mathbb{R}$ are linked by the ODE:

$$
\left\{\begin{array}{l}
y^{\prime}(t)=(A+v(t) B) y(t), \forall t \in(0, T) \\
y(0)=y_{0}
\end{array}\right.
$$

the initial condition $y_{0}$ being fixed.

Given two controls $v$ and $\widetilde{v}$ and the corresponding states $y$ and $\widetilde{y}$, we first note that:

$$
\begin{aligned}
J(\widetilde{v})-J(v)= & (\widetilde{y}(T)-y(T)) \cdot C(\widetilde{y}(T)-y(T))+2(\widetilde{y}(T)-y(T)) \cdot C y(T) \\
& -\alpha \int_{0}^{T}(\widetilde{v}(t)-v(t))(\widetilde{v}(t)+v(t)) d t .
\end{aligned}
$$

We then introduce an auxiliary function $z:[0, T] \rightarrow \mathbb{R}^{n}$ associated to $y$ and $v$ by

$$
\left\{\begin{array}{l}
z^{\prime}(t)=-\left(A^{*}+v(t) B^{*}\right) z(t) \\
z(T)=C y(T)
\end{array}\right.
$$

where $A^{*}$ and $B^{*}$ are the transposed matrices of $A$ and $B$.

Focusing on the second term of the right hand side of this equation, we get:

$$
(\widetilde{y}(T)-y(T)) \cdot C y(T)=\int_{0}^{T}(\widetilde{v}(t)-v(t)) B \widetilde{y}(t) \cdot z(t) d t .
$$

Thus, we finally obtain:

$J(\widetilde{v})-J(v)=(\widetilde{y}(T)-y(T)) \cdot C(\widetilde{y}(T)-y(T))+\alpha \int_{0}^{T}(\widetilde{v}(t)-v(t))\left(\frac{2}{\alpha} B \widetilde{y}(t) \cdot z(t)-\widetilde{v}(t)-v(t)\right) d t$.

A simple way to guarantee that $\widetilde{v}$ gives a better cost functional value than $v$, is to impose that:

$$
(\widetilde{v}(t)-v(t))\left(\frac{2}{\alpha} B \widetilde{y}(t) \cdot z(t)-\widetilde{v}(t)-v(t)\right) \geq 0 .
$$

Following this approach, the sequence $\left(v^{k}\right)_{k \in \mathbb{N}}$ defined iteratively by the implicit equation $v^{k+1}=\frac{1}{\alpha} B y^{k+1}(t) \cdot z^{k}(t)$, where $y^{k+1}$ and $z^{k}$ correspond to $v^{k+1}$ and $v^{k}$ respectively, optimizes $J$ monotonically since

$J\left(v^{k+1}\right)-J\left(v^{k}\right)=\left(y^{k+1}(T)-y^{k}(T)\right) \cdot C\left(y^{k+1}(T)-y^{k}(T)\right)+\alpha \int_{0}^{T}\left(v^{k+1}(t)-v^{k}(t)\right)^{2} d t \geq 0$.

In this article, we prove the convergence of generalizations of this algorithm towards a critical point of $J$ in the case of the Schrödinger partial differential equation:

$$
i \partial_{t} \psi(x, t)-[H-\mu(x) \varepsilon(t)] \psi(x, t)=0 .
$$

This equation governs the evolution of a quantum system, described by its wave function $\psi$, that interacts with a laser pulse of amplitude $\varepsilon$, the control variable. The factor $\mu$ is 
the dipole moment operator of the system. In what follows, $H=-\Delta+V$ where $\Delta$ is the Laplacian operator and $V=V(x)$ the electrostatic potential in which the system evolves. We refer to [22] for more details about models involved in quantum control.

The paper is organized as follows: we start in Section 2 with some necessary results about the linear and nonlinear Schrödinger equations involved in the problem we are considering. We then present the optimization problem in Section 3, and claim some regularity results about the corresponding cost functional in Section 4. We introduce in Section 5 an important tool for proving the convergence of the sequence, namely the Eojasiewicz inequality and some of its generalizations. The definition of the monotonically optimizing sequence is given in Section 6 where some useful properties are also claimed. The convergence of the sequences is proved in Section 7 and a first result about their rate of convergence follows in the last section.

Throughout this paper, $T$ is a positive real number representing the time of control of a physico-chemical process. We denote by $L^{2}$ and $L^{\infty}$ the spaces $L^{2}\left(\mathbb{R}^{3}, \mathbb{C}\right)$ and $L^{\infty}\left(\mathbb{R}^{3}, \mathbb{C}\right)$, $W^{p, \infty}\left(\mathbb{R}^{3}, \mathbb{R}\right)$ with $p \in[1,+\infty)$ by $W^{p, \infty}$, the Sobolev space $H^{2}\left(\mathbb{R}^{3}, \mathbb{C}\right)$ by $H^{2}$ and $L^{p}(0, T ; X)$, with $p \in[1,+\infty)$ denotes the usual Lebesgue space taking its values in a Banach space $X$. We also use the notation $\langle.||$.$\rangle and \langle.,$.$\rangle defined by:$

$$
\langle f|A| g\rangle=\int_{\mathbb{R}^{3}} \overline{f(x)} A g(x) d x,\langle f, g\rangle=\int_{\mathbb{R}^{3}} \overline{f(x)} g(x) d x,
$$

where $f$ and $g$ are in $L^{2}$ and $A$ is an operator on $L^{2}$. To simplify our notation, the space variable $x$ will often be omitted. Finally, for $\left.\left.h \in L^{p}(0, T ; X), p \in\right] 1, \infty\right]$, we recall that $\|h\|_{L^{p}(0, T ; X)}=\|t \mapsto\| h(t)\left\|_{X}\right\|_{L^{p}(0, T)}$. Finally, we denote by $\operatorname{Im}(z)$ and $\operatorname{Re}(z)$ the imaginary and the real part of a complex number $z$.

\section{Preliminary existence results}

The sequences we study in this paper are defined through iterative resolutions of Schrödinger equations. Before introducing the relevant framework of our study, we present here some necessary preliminary existence and regularity results concerning these equations. The first one will correspond later to the initialization step in the definition of the sequences. This lemma is a corollary of a general result on time dependent hamiltonians (see [23], p285, Theorem X.70) but for the sake of clarity, we present here an approach using other techniques also useful in the proof of the next lemmas.

Lemma 1 Let $\mu$ and $V$ belong to $W^{2, \infty}$ and let $H=-\Delta+V$. If $\varepsilon \in L^{2}(0, T)$ and $\psi_{0} \in H^{2}$, the equation

$$
\left\{\begin{array}{l}
i \partial_{t} \psi(x, t)-[H(x)-\mu(x) \varepsilon(t)] \psi(x, t)=0 \\
\psi(x, 0)=\psi_{0}(x)
\end{array}\right.
$$

has a unique solution $\psi \in L^{\infty}\left(0, T ; H^{2}\right) \cap W^{1, \infty}\left(0, T ; L^{2}\right)$. Moreover:

$$
\forall t \in[0, T], \quad\|\psi(t)\|_{L^{2}}=\left\|\psi_{0}\right\|_{L^{2}} .
$$

Proof: One can also read a similar proof in [3] but we give here some details. It is well known (see [11] for instance) that for any $T>0$ and $u_{0} \in H^{2}$, the Schrödinger equation

$$
\left\{\begin{array}{l}
i \partial_{t} u(x, t)+\Delta u(x, t)=0, \quad x \in \mathbb{R}, t \in[0, T] \\
u(x, 0)=u_{0}(x), \quad x \in \mathbb{R}
\end{array}\right.
$$

has a unique solution $u(t)=S(t) u_{0}$ such that $u \in C\left([0, T] ; H^{2}\right) \cap C^{1}\left([0, T] ; L^{2}\right)$, where $(S(t))_{t \in \mathbb{R}}$ denotes the free Schrödinger semi-group e ${ }^{i t \Delta}$. Moreover, for all $t \in[0, T]$ we have 


$$
\|u(t)\|_{H^{2}}=\left\|S(t) u_{0}\right\|_{H^{2}}=\left\|u_{0}\right\|_{H^{2}} .
$$

Let $\lambda>0$ be a given positive number which will be fixed hereafter and denote by $Y$ the space $C\left([0, T] ; H^{2}\right)$ endowed with the norm $\|\psi\|_{Y}=\sup _{t \in[0, T]} \mathrm{e}^{-\lambda t}\|\psi(t)\|_{H^{2}}$. The solution of equation (2) is obtained equivalently as a solution to the integral equation

$$
\psi(t)=S(t) \psi_{0}+i \int_{0}^{t} S(t-s) W(s) \psi(s) d s
$$

where $W(x, t)=-V(x)+\mu(x) \varepsilon(t)$ for all $t \in[0, T], x \in \mathbb{R}^{3}$. We are going to show that this equation has a unique solution in $Y$, by proving that operator $\Phi$ defined by

$$
\Phi(\psi)(t)=S(t) \psi_{0}+i \int_{0}^{t} S(t-s) W(s) \psi(s) d s
$$

has a unique fixed point in a closed ball $B_{R}=\left\{\psi \in Y ;\|\psi\|_{Y} \leq R\right\}$ for suitable $R$.

If $\psi \in B_{R}$, then $\|\psi(s)\|_{H^{2}} \leq \mathrm{e}^{\lambda s}\|\psi\|_{Y} \leq R \mathrm{e}^{\lambda s}$ and since $W \in L^{2}\left(0, T ; W^{2, \infty}\right)$, we can set $\rho>0$ such that $\|W\|_{L^{2}\left(0, T ; W^{2, \infty}\right)} \leq \rho$. Using estimate (4) and Cauchy-Schwarz inequality we obtain

$$
\|\Phi(\psi)(t)\|_{H^{2}} \leq\left\|\psi_{0}\right\|_{H^{2}}+\int_{0}^{t}\|W(s) \psi(s)\|_{H^{2}} d s \leq\left\|\psi_{0}\right\|_{H^{2}}+\rho R\left(\int_{0}^{t} \mathrm{e}^{2 \lambda s} d s\right)^{\frac{1}{2}} .
$$

It follows that if $R>0$ is large enough so that $\left\|\psi_{0}\right\|_{H^{2}} \leq \frac{R}{2}$ and if we choose $\lambda>2 \rho^{2}$, then

$$
\|\Phi(\psi)\|_{Y} \leq \sup _{t \in[0, T]} \mathrm{e}^{-\lambda t}\left\|\psi_{0}\right\|_{H^{2}}+\rho R\left(\int_{0}^{t} \mathrm{e}^{2 \lambda(s-t)} d s\right)^{\frac{1}{2}} \leq \frac{R}{2}+\frac{\rho R}{\sqrt{2 \lambda}} \leq R .
$$

This means that $\Phi$ maps $B_{R}$ into itself. Then, for $\psi_{1}, \psi_{2} \in B_{R}$ it is clear that

$$
\left\|\left(\Phi\left(\psi_{1}\right)-\Phi\left(\psi_{2}\right)\right)(t)\right\|_{H^{2}} \leq \int_{0}^{t}\left\|W(s)\left(\psi_{1}-\psi_{2}\right)(s)\right\|_{H^{2}} d s \leq \rho\left\|\psi_{1}-\psi_{2}\right\|_{Y}\left(\frac{\mathrm{e}^{2 \lambda t}-1}{2 \lambda}\right)^{\frac{1}{2}},
$$

and since $\lambda$ has been appropriately chosen, this proves that $\Phi$ is a strict contraction from $B_{R}$ into itself as

$\left\|\left(\Phi\left(\psi_{1}\right)-\Phi\left(\psi_{2}\right)\right)\right\|_{Y} \leq \rho\left\|\psi_{1}-\psi_{2}\right\|_{Y} \sup _{t \in[0, T]}\left(\frac{1-\mathrm{e}^{-2 \lambda t}}{2 \lambda}\right)^{\frac{1}{2}} \leq \frac{\rho}{\sqrt{2 \lambda}}\left\|\psi_{1}-\psi_{2}\right\|_{Y} \leq \frac{1}{2}\left\|\psi_{1}-\psi_{2}\right\|_{Y}$

and therefore $\Phi$ has a unique fixed point, yielding the solution of equation (2) in $L^{\infty}\left(0, T ; H^{2}\right)$. One can notice that uniqueness is not only true in $B_{R}$ but also easily proved using the norm in $L^{\infty}\left(0, T ; L^{2}\right)$. Moreover, calculating $\operatorname{Im} \int_{\mathbb{R}}(2) \cdot \bar{\psi}(x) d x$, one can prove the conservation of the $L^{2}$-norm (3) and finally, using equation (2), it is easy to obtain that $\psi \in W^{1, \infty}\left(0, T ; L^{2}\right)$.

We will also have recourse to a similar lemma, dealing with equation (2) with a non zero source term.

Lemma 2 Let $H, \mu, \varepsilon$ be as above and $\psi \in L^{\infty}\left(0, T ; H^{2}\right)$. Given $\varepsilon^{\prime} \in L^{2}(0, T)$, the equation:

$$
\left\{\begin{array}{l}
i \partial_{t} \psi^{\prime}(x, t)-[H(x)-\mu(x) \varepsilon(t)] \psi^{\prime}(x, t)=-\mu(x) \varepsilon^{\prime}(t) \psi(t, x) \\
\psi^{\prime}(x, 0)=0
\end{array}\right.
$$


has a unique solution $\psi^{\prime} \in L^{\infty}\left(0, T ; H^{2}\right) \cap W^{1, \infty}\left(0, T ; L^{2}\right)$. Moreover the following estimate holds:

$$
\left\|\psi^{\prime}\right\|_{L^{\infty}\left(0, T ; L^{2}\right)} \leq 2\|\mu\|_{L^{\infty}}\left\|\varepsilon^{\prime}\right\|_{L^{1}(0, T)}\|\psi\|_{L^{\infty}\left(0, T ; L^{2}\right)} .
$$

Proof: The key point to prove the existence of a solution for $(5)$ is to underline the fact that the source term $f(x, t)=\mu(x) \varepsilon^{\prime}(t) \psi(t, x)$ of this linear Schrödinger equation belongs to $L^{2}\left(0, T ; H^{2}\right)$. It is then very classical to get from the Lemma 1 the existence and uniqueness of a solution $\psi^{\prime}$ to equation (5) in $L^{\infty}\left(0, T ; H^{2}\right) \cap W^{1, \infty}\left(0, T ; L^{2}\right)$. Consider now $\varphi \in C([0, T])$ defined on $[0, T]$ by $\varphi(t)=\left\|\psi^{\prime}(t)\right\|_{L^{2}}^{2}$. We have:

$$
\frac{d}{d t} \varphi(t)=2 \operatorname{Re}\left\langle\psi^{\prime}(t), \frac{H-\mu \varepsilon(t)}{i} \psi^{\prime}(t)-\frac{\mu \varepsilon^{\prime}(t)}{i} \psi(t)\right\rangle=-2 \varepsilon^{\prime}(t) \operatorname{Im}\left\langle\psi^{\prime}(t)|\mu| \psi(t)\right\rangle .
$$

Moreover, there exists $t_{0}$ such that: $\varphi\left(t_{0}\right)=\sup _{t \in[0, T]}\left\{\left\|\psi^{\prime}(t)\right\|_{L^{2}}^{2}\right\}$. We suppose $\varepsilon^{\prime} \neq 0$ and $\psi \neq 0$, so that $\varphi\left(t_{0}\right) \neq 0$ by uniqueness of the solution of $(5)$. Since $\psi^{\prime}(x, 0)=0$ for all $x \in \mathbb{R}^{3}$, the integration of (7) between 0 and $t_{0}$ yields $\varphi\left(t_{0}\right)=\int_{0}^{t_{0}}-2 \varepsilon^{\prime}(t) \operatorname{Im}\left\langle\psi^{\prime}(t)|\mu| \psi(t)\right\rangle d t$, then:

$$
\varphi\left(t_{0}\right)=\left\|\psi^{\prime}\left(t_{0}\right)\right\|_{L^{2}}^{2} \leq\|\mu\|_{L^{\infty}}\left\|\psi^{\prime}\left(t_{0}\right)\right\|_{L^{2}} \int_{0}^{T} 2 \mid \varepsilon^{\prime}(t)\|\psi(t)\|_{L^{2}} d t .
$$

Since $\left\|\psi^{\prime}(t)\right\|_{L^{2}} \leq\left\|\psi^{\prime}\left(t_{0}\right)\right\|_{L^{2}}$ for all $t \in[0, T]$, we obtain

$$
\left\|\psi^{\prime}(t)\right\|_{L^{2}} \leq\left\|\psi^{\prime}\left(t_{0}\right)\right\|_{L^{2}} \leq 2\|\mu\|_{L^{\infty}}\|\psi\|_{L^{\infty}\left(0, T ; L^{2}\right)} \int_{0}^{T}\left|\varepsilon^{\prime}(t)\right| d t,
$$

what ends the proof of estimate (6).

Finally, we claim a last result that will be useful to tackle the problems related to a nonlinear Schrödinger equation we encounter in this study. Actually the nonlinearity we consider here is the one that appears naturally in the adjoint system from a quadratic cost functional (as $J$ is in (14)), even when the state equation is linear.

Lemma 3 Let $H, \mu, \varepsilon$ and $\psi_{0}$ defined as above. Given $\chi \in L^{\infty}\left(0, T ; H^{2}\right)$, the nonlinear Schrödinger equation:

$$
\left\{\begin{array}{l}
i \partial_{t} \psi(x, t)-[H(x)-\mu(x) \varepsilon(t)+\operatorname{Im}\langle\chi(t)|\mu| \psi(t)\rangle \mu(x)] \psi(x, t)=0 \\
\psi(x, 0)=\psi_{0}(x)
\end{array}\right.
$$

has a unique solution $\psi \in L^{\infty}\left(0, T ; H^{2}\right) \cap W^{1, \infty}\left(0, T ; L^{2}\right)$.

Proof: - First Step -

Let $u$ and $\chi \in H^{2}$, we denote the nonlinear term by $F(u)=\operatorname{Im}\langle\chi|\mu| u\rangle \mu u$ and we can prove that one has the following estimates: $\exists C=C(\chi, \mu)>0$ such that

$$
\begin{aligned}
\forall u, v \in L^{2}, \quad\|F(u)-F(v)\|_{L^{2}} & \leq C\left(\|u\|_{L^{2}}+\|v\|_{L^{2}}\right)\|u-v\|_{L^{2}} \\
\forall u, v \in H^{2}, \quad\|F(u)-F(v)\|_{H^{2}} & \leq C\left(\|u\|_{L^{2}}+\|v\|_{H^{2}}\right)\|u-v\|_{H^{2}} \\
\|F(u)\|_{H^{2}} & \leq C\|u\|_{L^{2}}\|u\|_{H^{2}}
\end{aligned}
$$

Indeed

$$
\begin{aligned}
\|F(u)-F(v)\|_{L^{2}} & \leq\|\operatorname{Im}\langle\chi|\mu| u\rangle \mu u-\operatorname{Im}\langle\chi|\mu| v\rangle \mu v\|_{L^{2}} \\
& \leq\|\operatorname{Im}\langle\chi|\mu| u\rangle \mu(u-v)\|_{L^{2}}+\|\operatorname{Im}\langle\chi|\mu|(u-v)\rangle \mu v\|_{L^{2}} \\
& \leq\|\mu\|_{L^{\infty}}^{2}\|\chi\|_{L^{2}}\left(\|u\|_{L^{2}}+\|v\|_{L^{2}}\right)\|u-v\|_{L^{2}}
\end{aligned}
$$


which proves (9). Now, we have to establish (10) and (11). First of all we have

$$
\|F(u)-F(v)\|_{H^{2}}^{2}=\|F(u)-F(v)\|_{L^{2}}^{2}+\|\Delta F(u)-\Delta F(v)\|_{L^{2}}^{2} .
$$

The first term of the right hand side is conveniently bounded in (9). Moreover

$$
\begin{aligned}
\|\Delta F(u)-\Delta F(v)\|_{L^{2}} & \leq\|\operatorname{Im}\langle\chi|\mu| u-v\rangle \Delta(\mu(u-v))\|_{L^{2}}+\|\operatorname{Im}\langle\chi|\mu| v\rangle \Delta(\mu v)\|_{L^{2}} \\
& \leq\|\mu\|_{W^{2, \infty}}^{2}\|\chi\|_{L^{2}}\left(\|u\|_{L^{2}}+\|v\|_{H^{2}}\right)\|u-v\|_{H^{2}} \\
& \leq C\left(\|u\|_{L^{2}}+\|v\|_{H^{2}}\right)\|u-v\|_{H^{2}} .
\end{aligned}
$$

Then, $F$ is locally lipschitz in $H^{2}$. Therefore, taking $v=0$, we also get (11).

- Second Step -

The proof of a local-in-time result is based again on a fixed point theorem. We begin by fixing an arbitrary time $T>0$ and considering $\tau \in] 0, T]$. We also consider the functional

$$
\xi: \psi \longmapsto U(., 0) \psi_{0}-i \int_{0}^{\cdot} U(., s) F(\psi(s)) d s,
$$

where $\{U(t, s), s, t \in[0, T]\}$ is the propagator associated with the operator $H-\mu \varepsilon$ and induced by Lemma 1 (such that $U(t, s) \in \mathcal{L}\left(H^{2}\right)$ - for details, see [5]), and the set

$$
B=\left\{v \in L^{\infty}\left(0, \tau ; H^{2}\right),\|\psi\|_{L^{\infty}\left(0, \tau ; H^{2}\right)} \leq 2 M\left\|\psi_{0}\right\|_{H^{2}}\right\} .
$$

where $M$ satisfies $\forall v \in H^{2},\|U(t, s) v\|_{H^{2}} \leq M\|v\|_{H^{2}}$.

If $\tau>0$ is small enough, the functional $\xi$ maps $B$ into itself and is a strict contraction in the Banach space $L^{\infty}\left(0, \tau ; H^{2}\right)$. Indeed, on the one hand, from estimate (11), if $\psi \in B$, we have for all $t \in[0, \tau]$ :

$$
\begin{aligned}
\|\xi(\psi)(t)\|_{H^{2}} & \leq\left\|U(t, 0) \psi_{0}-i \int_{0}^{t} U(t, s) F(\psi(s)) d s\right\|_{H^{2}} \\
& \leq M\left\|\psi_{0}\right\|_{H^{2}}+\tau M\|F(\psi)\|_{L^{\infty}\left(0, \tau ; H^{2}\right)} \\
& \leq M\left\|\psi_{0}\right\|_{H^{2}}+\tau C M\|\psi\|_{L^{\infty}\left(0, \tau ; L^{2}\right)}\|\psi\|_{L^{\infty}\left(0, \tau ; H^{2}\right)} \\
& \leq M\left\|\psi_{0}\right\|_{H^{2}}+4 \tau C M^{3}\left\|\psi_{0}\right\|_{H^{2}}^{2} .
\end{aligned}
$$

Then, if we choose $\tau$ such that $4 \tau C M^{2}\left\|\psi_{0}\right\|_{H^{2}}<1$ we obtain $\|\xi(\psi)\|_{L^{\infty}\left(0, \tau ; H^{2}\right)} \leq 2 M\left\|\psi_{0}\right\|_{H^{2}}$ and $\xi(\psi)$ belongs to $B$. On the other hand, if $\psi_{1}$ and $\psi_{2} \in B$, then for all $t$ in $[0, \tau]$ we have,

$$
\begin{aligned}
\left\|\xi\left(\psi_{1}\right)(t)-\xi\left(\psi_{2}\right)(t)\right\|_{H^{2}} & =\left\|\int_{0}^{t} U(t, s)\left(F\left(\psi_{1}(s)\right)-F\left(\psi_{2}(s)\right)\right) d s\right\|_{H^{2}} \\
& \leq C M\left(\left\|\psi_{1}\right\|_{L^{\infty}\left(0, \tau ; L^{2}\right)}+\left\|\psi_{2}\right\|_{L^{\infty}\left(0, \tau ; H^{2}\right)}\right) \int_{0}^{t}\left\|\psi_{1}(s)-\psi_{2}(s)\right\|_{H^{2}} d s \\
& \leq 4 \tau C M^{2}\left\|\psi_{0}\right\|_{H^{2}}\left\|\psi_{1}-\psi_{2}\right\|_{L^{\infty}\left(0, \tau ; H^{2}\right)}
\end{aligned}
$$

with $4 \tau C M^{2}\left\|\psi_{0}\right\|_{H^{2}}<1$. Therefore, from a usual fixed point theorem, we can deduce existence and uniqueness in the set $B$, then in $L^{\infty}\left(0, \tau ; H^{2}\right)$, for $\tau>0$ small enough, of the solution of equation

$$
\psi(t)=U(t, 0) \psi_{0}-i \int_{0}^{t} U(t, s) F(\psi(s)) d s
$$

which is in fact equivalent to equation (8). Moreover, using (8), it is easy to prove that $\partial_{t} \psi$ belongs to $L^{\infty}\left(0, \tau ; L^{2}\right)$. 
The last point is then to prove the uniqueness of the solution $u$ of (8) in the space $L^{\infty}\left(0, \tau ; H^{2}\right) \cap W^{1, \infty}\left(0, \tau ; L^{2}\right)$. Let $\psi_{1}$ and $\psi_{2}$ be two solutions of (8) and $w$ equal to $\psi_{1}-\psi_{2}$. Then $w(0)=0$ and

$$
i \partial_{t} w-[H(x)-\mu(x) \varepsilon(t)] w=F\left(\psi_{2}\right)-F\left(\psi_{1}\right) .
$$

Calculating $\operatorname{Im} \int_{\mathbb{R}}(13) \cdot \bar{w}(x) d x$ and using (9) we obtain $\frac{d}{d t}\left(\|w\|_{L^{2}}^{2}\right) \leq C\|w\|_{L^{2}}^{2}$ and uniqueness follows by Gronwall lemma. Hence the proof of uniqueness, existence and regularity of the solution of equation (8) in $\mathbb{R} \times[0, \tau]$ for any time $\tau<\frac{1}{4 C M^{2}\left\|\psi_{0}\right\|_{H^{2}}}$.

- Third Step -

Now, the goal is to obtain an a priori estimate of the solution in $W^{1, \infty}\left(0, T ; L^{2}\right) \cap L^{\infty}\left(0, T ; H^{2}\right)$ for any arbitrary time $T$, in order to prove that the local solution we obtained previously exists globally because we have a uniform bound on the norm $\|\psi(t)\|_{H^{2}}+\left\|\partial_{t} \psi(t)\right\|_{L^{2}}$.

Actually, since equation (8) is equivalent to the integral equation (12) and since it is easy to prove the conservation of the $L^{2}$-norm of the solution, we have,

$$
\begin{aligned}
\|\psi(t)\|_{H^{2}} & \leq\left\|U(t, 0) \psi_{0}\right\|_{H^{2}}+\left\|\int_{0}^{t} U(t, s) F(\psi(s)) d s\right\|_{H^{2}} \\
& \leq M\left\|\psi_{0}\right\|_{H^{2}}+M C \int_{0}^{t}\|\psi(s)\|_{L^{2}}\|\psi(s)\|_{H^{2}} d s \\
& \leq C_{0, T}\left(1+\int_{0}^{t}\|\psi(s)\|_{H^{2}} d s\right)
\end{aligned}
$$

where $C_{0, T}>0$ is a generic constant depending on the time $T$, on $\mu, \chi$ and on $\left\|\psi_{0}\right\|_{H^{2}}$. We finally obtain from Gronwall lemma and from equation (8), that $\|\psi(t)\|_{H^{2}}+\left\|\partial_{t} \psi(t)\right\|_{L^{2}} \leq C_{0, T}$ for all $t \in[0, T]$. Hence the proof of Lemma 3 .

\section{Optimization problem}

Let us now present the optimization problem we are dealing with in this paper. Let $O$ be a positive symmetric bounded operator on $H^{2}$ and $\alpha$ and $T$ two positive real numbers. Given $\psi_{0} \in H^{2}$, we consider the cost functional $J$ defined on $L^{2}(0, T)$ by:

$$
J(\varepsilon)=\langle\psi(T)|O| \psi(T)\rangle-\alpha \int_{0}^{T} \varepsilon^{2}(t) d t,
$$

where $\psi$ is the solution of (2). In all the sequel we suppose that $\left\|\psi_{0}\right\|_{L^{2}}=1$. The existence of a minimizer for similar cost functionals (with the opposite sign) has been obtained in [3], [4] and [9] and follows from the construction of a minimizing sequence and a compactness lemma (Aubin's lemma). Here, the point is to maximize the functional $J$ and as usual, at the maximum of $J$, the Euler-Lagrange critical point equations are satisfied. A standard way to write these equations is to use a Lagrange multiplier $\chi(x, t)$ usually called adjoint state. The following critical point equations are thus obtained, for $x \in \mathbb{R}^{3}$ and $t \in(0, T)$ :

$$
\begin{aligned}
& \left\{\begin{array}{l}
i \partial_{t} \psi(x, t)-[H(x)-\mu(x) \varepsilon(t)] \psi(x, t)=0, \\
\psi(x, 0)=\psi_{0}(x),
\end{array}\right. \\
& \left\{\begin{array}{l}
i \partial_{t} \chi(x, t)-[H(x)-\mu(x) \varepsilon(t)] \chi(x, t)=0, \\
\chi(x, T)=O \psi(x, T),
\end{array}\right.
\end{aligned}
$$




$$
\alpha \varepsilon(t)+\operatorname{Im}\langle\psi(t)|\mu| \chi(t)\rangle=0 .
$$

The existence of $\chi \in L^{\infty}\left(0, T ; H^{2}\right)$ results from an adaptation of Lemma 1 , as for $\psi(T) \in H^{2}$ since equation (15) is actually equation (2). In what follows, we also consider the linearized equation of (16):

$$
\left\{\begin{array}{l}
i \partial_{t} \chi^{\prime}(x, t)-[H(x)-\mu(x) \varepsilon(t)] \chi^{\prime}(x, t)=-\mu(x) \varepsilon^{\prime}(t) \chi(t, x) \\
\chi^{\prime}(x, T)=O \psi^{\prime}(T)
\end{array}\right.
$$

where $\varepsilon^{\prime} \in L^{2}(0, T)$ and $\psi^{\prime}$ is the solution of (5), corresponding to the solution $\psi$ of (15). The existence of $\chi^{\prime} \in L^{\infty}\left(0, T ; H^{2}\right)$ follows from Lemma 2. The analysis done in the proof of estimate (6) gives in this case:

$$
\left\|\chi^{\prime}(t)\right\|_{L^{2}} \leq 2\|\mu\|_{L^{\infty}}\left\|\varepsilon^{\prime}\right\|_{L^{1}(0, T)}\|\chi\|_{L^{\infty}\left(0, T ; L^{2}\right)}+\left\|\chi^{\prime}(T)\right\|_{L^{2}} .
$$

Since $\chi(T)=O \psi(T), \chi^{\prime}(T)=O \psi^{\prime}(T)$ and from (6) and the conservation of the $L^{2}$-norm, we obtain

$$
\begin{aligned}
\left\|\chi^{\prime}\right\|_{L^{\infty}\left(0, T ; L^{2}\right)} & \leq 2\|\mu\|_{L^{\infty}}\left\|\varepsilon^{\prime}\right\|_{L^{1}(0, T)}\|\chi\|_{L^{\infty}\left(0, T ; L^{2}\right)}+2\|O\|_{*}\|\mu\|_{L^{\infty}}\left\|\varepsilon^{\prime}\right\|_{L^{1}(0, T)}\|\psi\|_{L^{\infty}\left(0, T ; L^{2}\right)} \\
& \leq 4\|\mu\|_{L^{\infty}}\|O\|_{*}\left\|\varepsilon^{\prime}\right\|_{L^{1}(0, T)},
\end{aligned}
$$

where $\|O\|_{*}$ denotes the operator norm of $O$ on $L^{2}$.

\section{Properties of the functional $J$}

We begin with some properties about the regularity of the cost functional $J$.

\subsection{Gradient of $J$}

We start with some first order properties. As often, the use of the adjoint state $\chi$ allows us to simplify the computation of the derivative of $J$. This result is the purpose of the next lemma.

Lemma 4 The cost functional $J$ is differentiable on $L^{2}(0, T)$ and its gradient can be expressed by

$$
\left(\nabla J(\varepsilon), \varepsilon^{\prime}\right)=-2 \int_{0}^{T}(\alpha \varepsilon(t)+\operatorname{Im}\langle\chi(t)|\mu| \psi(t)\rangle) \varepsilon^{\prime}(t) d t,
$$

where $(\cdot, \cdot)$ is the usual inner product on $L^{2}(0, T)$ and $\psi$ and $\chi$ are the solutions of (15) and (16).

Proof: We only give here a sketch of the proof. The details can be found in reference [3] for a slightly different cost functional. The main point is to prove the differentiability of the functional $\phi: \varepsilon \in L^{2}(0, T) \mapsto \psi(T)$, where $\psi$ is the solution of equation (15). Actually, one can prove that the solution $\psi^{\prime}$ of (5) is such that $D \phi(\varepsilon)\left[\varepsilon^{\prime}\right]=\psi^{\prime}(T)$. Therefore, since $J(\varepsilon)=\langle\psi(T)|O| \psi(T)\rangle-\alpha \int_{0}^{T} \varepsilon^{2}(t) d t$, we have

$$
\left(\nabla J(\varepsilon), \varepsilon^{\prime}\right)=2 \operatorname{Re}\left\langle\psi^{\prime}(T)|O| \psi(T)\right\rangle-2 \alpha \int_{0}^{T} \varepsilon(t) \varepsilon^{\prime}(t) d t .
$$

To end the proof of (20), we consider the solution $\chi$ of the adjoint state equation (16) and we multiply equation (5) by $\bar{\chi}$ (the complex conjugate of $\chi$ ), integrate on $\mathbb{R} \times[0, T]$ and take the imaginary part. We obtain:

$$
\operatorname{Im} \int_{0}^{T} \int_{\mathbb{R}}\left(i \partial_{t} \psi^{\prime}-[H-\mu \varepsilon] \psi^{\prime}\right) \bar{\chi}=\operatorname{Im} \int_{0}^{T} \int_{\mathbb{R}} \mu \varepsilon^{\prime} \psi \bar{\chi}
$$


After an integration by parts and since $\psi^{\prime}(0)=0$, we get

$$
\operatorname{Im} \int_{0}^{T} \int_{\mathbb{R}} \overline{i \partial_{t} \chi} \psi^{\prime}-\operatorname{Im} \int_{\mathbb{R}} \psi^{\prime}(T) \overline{i \chi(T)}-\operatorname{Im} \int_{0}^{T} \int_{\mathbb{R}} \overline{[H-\mu \varepsilon] \chi} \psi^{\prime}=\operatorname{Im} \int_{0}^{T} \int_{\mathbb{R}} \mu \varepsilon^{\prime} \psi \bar{\chi} .
$$

Since $\chi$ satisfies equation (16), we then obtain

$$
\operatorname{Re}\left\langle\psi^{\prime}(T)|O| \psi(T)\right\rangle=\operatorname{Re} \int_{\mathbb{R}} \psi^{\prime}(T) \overline{O \psi(T)}=-\operatorname{Im} \int_{0}^{T} \int_{\mathbb{R}} \mu \varepsilon^{\prime} \psi \bar{\chi}=-\int_{0}^{T} \operatorname{Im}\langle\chi(t)|\mu| \psi(t)\rangle \varepsilon^{\prime}(t) d t
$$

what ends the proof of the lemma.

In what follows, we denote by $\nabla J(\varepsilon)$ the function $t \mapsto-2(\alpha \varepsilon(t)+\operatorname{Im}\langle\chi(t)|\mu| \psi(t)\rangle)$ and by $C_{J}$ the set of the critical points of $J$, i.e.,

$$
C_{J}=\left\{\varepsilon \in L^{2}(0, T), \quad \forall t \in[0, T], \quad \alpha \varepsilon(t)+\operatorname{Im}\langle\chi(t)|\mu| \psi(t)\rangle=0\right\} .
$$

Note that, thanks to the results of the section 2, we have $C_{J} \subset L^{\infty}(0, T)$ since for all $\varepsilon \in C_{J}$,

$$
\|\varepsilon\|_{L^{\infty}(0, T)} \leq \frac{1}{\alpha}\|\langle\chi|\mu| \psi\rangle\|_{L^{\infty}(0, T)} \leq C\|\mu\|_{L^{\infty}}\|\chi\|_{L^{\infty}\left(0, T ; L^{2}\right)}\|\psi\|_{L^{\infty}\left(0, T ; L^{2}\right)} .
$$

Remark 1 : Note also that for $\alpha>6 T\|\mu\|_{L_{\infty}}^{2}\|O\|_{*}$, the set $C_{J}$ is reduced to one point. Indeed, suppose that $C_{J}$ contains two distinct points $\varepsilon_{1}$ and $\varepsilon_{2}$, we then have, for $t \in(0, T)$ :

$$
\alpha\left(\varepsilon_{2}(t)-\varepsilon_{1}(t)\right)+\operatorname{Im}\left\langle\chi_{2}(t)-\chi_{1}(t)|\mu| \psi_{2}(t)\right\rangle+\operatorname{Im}\left\langle\chi_{1}(t)|\mu| \psi_{2}(t)-\psi_{1}(t)\right\rangle=0,
$$

where $\psi_{1}, \psi_{2}$ (resp. $\left.\chi_{1}, \chi_{2}\right)$ are the solutions of (15) (resp. (16)) corresponding to $\varepsilon_{1}$ and $\varepsilon_{2}$ respectively. Using estimates (6) with $\psi=\psi_{1}, \psi^{\prime}=\psi_{2}-\psi_{1}, \varepsilon=\varepsilon_{2}$ and $\varepsilon^{\prime}=\varepsilon_{2}-\varepsilon_{1}$ and (18) with $\chi=\chi_{1}, \chi^{\prime}=\chi_{2}-\chi_{1}, \psi^{\prime}=\psi_{2}-\psi_{1}, \varepsilon=\varepsilon_{2}$ and $\varepsilon^{\prime}=\varepsilon_{2}-\varepsilon_{1}$, we obtain

$$
\alpha\left\|\varepsilon_{2}-\varepsilon_{1}\right\|_{L_{1}(0, T)} \leq 6 T\|\mu\|_{L^{\infty}}^{2}\|O\|_{*}\left\|\varepsilon_{2}-\varepsilon_{1}\right\|_{L^{1}(0, T)},
$$

which leads to $\alpha \leq 6 T\|O\|_{*}\|\mu\|_{L^{\infty}}^{2}$, and the result follows.

In order to prove the compactness of $C_{J}$, we introduce an important property of the application $\varepsilon(t) \mapsto \psi(x, t)$, firstly presented in a more general setting by J. M. Ball, J. E. Marsden and M. Slemrod in [2]. In our context, this result can be stated as follows.

Lemma 5 Assume that $\varepsilon \in L^{1}(0, T), \mu: X \rightarrow X$ is a bounded operator and that $H$ generates a $C^{0}$-semigroup of bounded linear operators on some Banach space $X$. For $x \in \mathbb{R}^{3}$ and $t \in(0, T)$, we denote by $\psi(x, t)$ the solution of

$$
\left\{\begin{array}{l}
i \partial_{t} \psi-[H-\mu \varepsilon] \psi=0 \\
\psi(0)=\psi_{0} \in X
\end{array}\right.
$$

Then, $\varepsilon \mapsto \psi$ is a compact mapping in the sense that for any weakly converging sequence $\left(\varepsilon_{n}\right)_{n \in \mathbb{N}}$ to $\varepsilon$ in $L^{1}(0, T),\left(\psi_{n}\right)_{n \in \mathbb{N}}$ converges strongly to $\psi$ in $C([0, T] ; X)$.

The precise proof of this result derives directly from [2] (Theorem 3.6, p580), see also [10] and [26]. It allows us to obtain the following lemma.

Lemma 6 For $\mu \in W^{2, \infty}, C_{J}$ is compact in $L^{\infty}(0, T)$. 
Proof: Consider a bounded sequence $\left(\varepsilon^{n}\right)_{n \in \mathbb{N}}$ of $C_{J}$. By definition, for all $n \in \mathbb{N}$, $\varepsilon^{n} \in L^{2}(0, T)$ and $\varepsilon^{n}(t)=-\frac{1}{\alpha}\left\langle\chi^{n}(t)|\mu| \psi^{n}(t)\right\rangle$ where $\psi^{n}$ and $\chi^{n}$ are the corresponding solutions of (15) and (16). It is also possible to extract a weakly convergent sub-sequence in $L^{2}(0, T)$, still denoted $\left(\varepsilon^{n}\right)_{n \in \mathbb{N}}$. From Lemma 1, one knows that the Hamiltonian $H=$ $-\Delta+V$ with $V \in W^{2, \infty}$ generates a $C^{0}$-semigroup of bounded linear operators on the Banach space $X=H^{2}$. Therefore, with $\mu \in W^{2, \infty}$ the conditions of Lemma 5 are fulfilled and we obtain the strong convergences $\psi^{n} \stackrel{n \rightarrow+\infty}{\longrightarrow} \psi$ and $\chi^{n} \stackrel{n \rightarrow+\infty}{\longrightarrow} \chi$ in $C\left([0, T] ; H^{2}\right)$. Thus, for

all $t \in(0, T), \int_{\mathbb{R}^{3}} \overline{\psi^{n}(t)} \mu \chi^{n}(t) d x \stackrel{n \rightarrow+\infty}{\longrightarrow} \int_{\mathbb{R}^{3}} \overline{\psi(t)} \mu \chi(t) d x$. The sequence $\left(\varepsilon_{n}(t)\right)_{n \in \mathbb{N}}$ then strongly converges in $L^{\infty}(0, T)$ and the result follows.

\subsection{Analyticity of $J$}

The implicit formulation of the derivative can be iteratively carried on in order to prove the analyticity of $J$.

Lemma 7 Let $\psi$ be the solution of (15) corresponding to $\varepsilon$. The functional

$$
\begin{aligned}
\vartheta: L^{2}(0, T) & \rightarrow L^{\infty}\left(0, T ; H^{2}\right) \cap W^{1, \infty}\left(0, T ; L^{2}\right) \\
\varepsilon & \mapsto \psi,
\end{aligned}
$$

is analytic.

Proof: Let $\varepsilon, \varepsilon^{\prime} \in L^{2}(0, T)$ be such that $\left\|\varepsilon^{\prime}\right\|_{L^{1}(0, T)} \leq \frac{1}{4\|\mu\|_{L^{\infty}}}$ and the sequence $\left(\psi^{\ell}\right)_{\ell \in \mathbb{N}} \in\left(L^{\infty}\left(0, T ; H^{2}\right)\right)^{\mathbb{N}}$ defined recursively by $\psi^{0}=\vartheta(\varepsilon)$ and for $\ell>0$ :

$$
\left\{\begin{array}{l}
i \partial_{t} \psi^{\ell}(x, t)-[H-\mu(x) \varepsilon(t)] \psi^{\ell}(x, t)=-\mu(x) \varepsilon^{\prime}(t) \psi^{\ell-1}(x, t) \\
\psi^{\ell}(x, 0)=0
\end{array}\right.
$$

The existence of $\psi^{\ell}$ is consequence of Lemma 2. Thanks to (6) applied with $\psi=\psi^{\ell-1}$ and $\psi^{\prime}=\psi^{\ell}$, one has for $\ell \geq 1$ and $t \in[0, T]$ :

$$
\left\|\psi^{\ell}(t)\right\|_{L^{2}} \leq 2\|\mu\|_{L^{\infty}}\left\|\varepsilon^{\prime}\right\|_{L^{1}(0, T)}\left\|\psi^{\ell-1}\right\|_{L^{\infty}\left(0, T ; L^{2}\right)} \leq 2^{\ell}\|\mu\|_{L^{\infty}}^{\ell}\left\|\varepsilon^{\prime}\right\|_{L^{1}(0, T)}^{\ell} .
$$

Given $N>0$, we obtain by summing (22) from $\ell=0$ to $N$ :

$$
\left\{\begin{array}{l}
i \partial_{t}\left(\sum_{\ell=0}^{N} \psi^{\ell}(x, t)\right)-\left[H-\mu(x)\left(\varepsilon(t)+\varepsilon^{\prime}(t)\right)\right]\left(\sum_{\ell=0}^{N} \psi^{\ell}(x, t)\right)=\mu(x) \varepsilon^{\prime}(t) \psi^{N}(x, t) \\
\sum_{\ell=0}^{N} \psi^{\ell}(x, 0)=\psi_{0}(x) .
\end{array}\right.
$$

On the other hand, one has:

$$
i \partial_{t} \vartheta\left(\varepsilon+\varepsilon^{\prime}\right)-\left[H-\mu(x)\left(\varepsilon(t)+\varepsilon^{\prime}(t)\right)\right] \vartheta\left(\varepsilon+\varepsilon^{\prime}\right)=0 .
$$

Subtracting (24) and (25) and using estimates (6) with $\psi=-\psi^{N}, \psi^{\prime}=\sum_{\ell=0}^{N} \psi^{\ell}(x, t)-\vartheta(\varepsilon+$ $\left.\varepsilon^{\prime}\right), \varepsilon=\varepsilon+\varepsilon^{\prime}$ and (23), we get:

$$
\left\|\vartheta\left(\varepsilon+\varepsilon^{\prime}\right)(t)-\sum_{\ell=0}^{N} \psi^{\ell}(t)\right\|_{L^{2}} \leq 2^{N}\|\mu\|_{L^{\infty}}^{N}\left\|\varepsilon^{\prime}\right\|_{L^{1}(0, T)}^{N} \leq 2^{-N}
$$


and the functional $\vartheta$ reads now: $\vartheta\left(\varepsilon+\varepsilon^{\prime}\right)=\sum_{\ell=0}^{\infty} \psi^{\ell}$ in $L^{2}(0, T)$. Since $\varepsilon^{\prime} \mapsto \psi^{\ell}$ is $\ell$-linear, the theorem follows.

The next lemma follows immediately from this result.

Lemma 8 The cost functional $J$ is analytic on $L^{2}(0, T)$.

\subsection{About the Hessian operator of $J$}

Let us now investigate some properties of the second order derivative of $J$. Though we express it as an implicit function of its argument $\varepsilon$, some results can be obtained from the next lemma.

Lemma 9 Let $\psi$ and $\chi$ be the solutions of (15) and (16). The functional $\gamma: \varepsilon \mapsto \operatorname{Im}\langle\chi|\mu| \psi\rangle$ is differentiable on $L^{2}(0, T)$ and one has:

$$
D \gamma(\varepsilon)\left[\varepsilon^{\prime}\right]=\operatorname{Im}\left\langle\chi^{\prime}|\mu| \psi\right\rangle+\operatorname{Im}\left\langle\chi|\mu| \psi^{\prime}\right\rangle,
$$

where $\psi^{\prime}$ and $\chi^{\prime}$ are the solutions of (5) and (17). Moreover, for all $\varepsilon \in L^{2}(0, T), D \gamma(\varepsilon)$ is compact on $L^{2}(0, T)$.

Proof: $\quad$ Let $\varepsilon \in L^{2}(0, T)$ and $\psi$ and $\chi$ the corresponding solutions of (15) and (16). As in the proof of Lemma 4 , the key-point is the differentiability of the functional $\vartheta$, defined in Lemma 7 on $L^{2}(0, T)$. Actually, $D \vartheta(\varepsilon)\left[\varepsilon^{\prime}\right]=\psi^{\prime}$, where $\psi^{\prime}$ is the solution of (5). The main explanations can be read in [4]. Repeating this argument for $\varepsilon \mapsto \chi$, we obtain that $\gamma$ is differentiable and we get $(26)$.

Let us now prove the compactness of this operator. Let $\left(\varepsilon^{\prime n}\right)_{n \in \mathbb{N}}$ be a bounded sequence in $L^{2}(0, T)$ and let $\left(\psi^{\prime n}\right)_{n \in \mathbb{N}}$ and $\left(\chi^{\prime n}\right)_{n \in \mathbb{N}}$ be the corresponding solutions of (5) and (17).

As $\psi^{\prime n} \in L^{\infty}\left(0, T ; H^{2}\right) \cap W^{1, \infty}\left(0, T ; L^{2}\right)$ (see the proof of Lemma 2), we have that $\psi^{\prime n} \in$ $C\left([0, T] ; L^{2}\right)$ and $\partial_{t} \psi^{\prime n} \in L^{2}\left(0, T ; L^{2}\right)$. By means of the continuity of:

$$
\begin{aligned}
L^{2}(0, T) & \rightarrow C\left([0, T] ; L^{2}\right) \\
\varepsilon^{\prime} & \mapsto \psi^{\prime}
\end{aligned} \text { and } \begin{aligned}
L^{2}(0, T) & \rightarrow L^{2}\left(0, T ; L^{2}\right) \\
\varepsilon^{\prime} & \mapsto \partial_{t} \psi^{\prime}
\end{aligned}
$$

there exist $\psi^{\prime \infty}$ such that, up to extraction, $\psi^{\prime n} \rightarrow \psi^{\prime \infty} \in L^{2}\left(0, T ; L^{2}\right)$ and

$$
\partial_{t} \psi^{\prime n} \rightarrow \partial_{t} \psi^{\prime \infty} \in L^{2}\left(0, T ; L^{2}\right) .
$$

Since $\psi^{\prime n}(0)=0$, we have $\psi^{\prime n}(t)=\int_{0}^{t} \partial_{t} \psi^{\prime n}(s) d s$ and (28) implies that for all $t \in[0, T]$, $\left(\left\|\psi^{\prime n}(t)\right\|_{L^{2}}\right)_{n \in \mathbb{N}}$ is uniformly bounded. Moreover, for all $t, t^{\prime} \in[0, T], t \leq t^{\prime}$, we have:

$$
\left\|\psi^{\prime n}\left(t^{\prime}\right)-\psi^{\prime n}(t)\right\|_{L^{2}} \leq \int_{t}^{t^{\prime}}\left\|\partial_{t} \psi^{\prime n}(s)\right\|_{L^{2}} d s \leq \sqrt{t^{\prime}-t}\left\|\partial_{t} \psi^{\prime n}\right\|_{L^{2}\left(0, T ; L^{2}\right)} .
$$

Combining this with (28), we find that $\left(\psi^{\prime n}\right)_{n \in \mathbb{N}}$ is an equicontinuous sequence in $C\left([0, T], L^{2}\right)$. We conclude by applying Ascoli's theorem to the family $\left\{\operatorname{Im}\left\langle\chi|\mu| \psi^{\prime n}\right\rangle, n \in \mathbb{N}\right\}$ of the space $C([0, T])$. Similar arguments apply for $\left\{\operatorname{Im}\left\langle\chi^{\prime n}|\mu| \psi\right\rangle, n \in \mathbb{N}\right\}$, and the results follows.

Thanks to the previous lemma, $J$ is twice differentiable and its Hessian operator reads:

$$
H_{J}(\varepsilon): \varepsilon^{\prime} \mapsto-2\left(\alpha \varepsilon^{\prime}+D \gamma(\varepsilon)\left[\varepsilon^{\prime}\right]\right) .
$$

In the sequel, a criterion ensuring that the Hessian operator of $J$ is invertible will be useful. The next lemma provides it. 
Lemma 10 Suppose that: $\alpha>6 T\|\mu\|_{L^{\infty}}^{2}\|O\|_{*}$. Then the operator $H_{J}(\varepsilon)$ is invertible on $L^{2}(0, T)$.

Proof: We keep the notation of Lemma 9. The Cauchy-Schwarz inequality, combined with (6) and (19) yields:

$$
\begin{aligned}
\left\|D \gamma(\varepsilon)\left[\varepsilon^{\prime}\right]\right\|_{L^{\infty}(0, T)} & \leq\|\mu\|_{L^{\infty}}\left(\left\|\chi^{\prime}\right\|_{L^{\infty}\left(0, T ; L^{2}\right)}+\|O\|_{*}\left\|\psi^{\prime}\right\|_{L^{\infty}\left(0, T ; L^{2}\right)}\right) \\
& \leq 6 \sqrt{T}\|\mu\|_{L^{\infty}}^{2}\|O\|_{*}\left\|\varepsilon^{\prime}\right\|_{L^{2}(0, T)} .
\end{aligned}
$$

Finally, thanks to the assumption of the lemma, one has

$$
\sup _{\left\{\varepsilon^{\prime},\left\|\varepsilon^{\prime}\right\|_{L^{2}(0, T)}=1\right\}}\left(\frac{1}{\alpha}\left\|D \gamma(\varepsilon)\left[\varepsilon^{\prime}\right]\right\|_{L^{2}(0, T)}\right)<1,
$$

which implies that $I+\frac{1}{\alpha} D \gamma(\varepsilon)$ is invertible and the result follows.

\section{5 Łojasiewicz inequality for the cost functional $J$}

Several convergence results of dynamical systems have been proved thanks to the Eojasiewicz inequality recalled here. In order to tackle the problem of the convergence of the optimizing sequence presented in the next section, we have to extend this inequality to the case of a compact set in an infinite dimensional space. The basic result considered in this section is the following ( $\mathrm{cf}[17,18])$ :

Theorem 1 Let $N$ be an integer and $\Gamma: \mathbb{R}^{N} \rightarrow \mathbb{R}$ be an analytic function in a neighborhood of a point $a \in \mathbb{R}^{N}$. Then there exists $\sigma>0$ and $\left.\left.\theta \in\right] 0, \frac{1}{2}\right]$ such that

$$
\forall x \in \mathbb{R}^{N},\|x-a\|<\sigma, \quad\|\nabla \Gamma(x)\| \geq|\Gamma(x)-\Gamma(a)|^{1-\theta},
$$

where $\|$.$\| is a given norm on \mathbb{R}^{N}$.

The real number $\theta$ is a Łojasiewicz exponent of $a$. Following the work [15] of M. A. Jendoubi (which simplifies the theorem of Łojasiewicz-Simon [28]), the latter theorem can be generalized to the case of infinite dimension.

Lemma 11 Given $\varepsilon \in L^{2}(0, T)$, there exists $\sigma^{\prime}>0, \kappa>0$ and $\left.\left.\theta^{\prime} \in\right] 0, \frac{1}{2}\right]$ such that:

$$
\forall \varepsilon^{\prime} \in L^{2}(0, T),\left\|\varepsilon^{\prime}-\varepsilon\right\|_{L^{2}(0, T)} \leq \sigma^{\prime}, \quad\left\|\nabla J\left(\varepsilon^{\prime}\right)\right\|_{L^{2}(0, T)} \geq \kappa\left|J\left(\varepsilon^{\prime}\right)-J(\varepsilon)\right|^{1-\theta^{\prime}} .
$$

We give the proof of this lemma in the appendix. A more precise result can be obtained if the Hessian operator under consideration is invertible at point $a$ (see e.g, [13]). Indeed, one can then show that $1 / 2$ is a Łojasiewicz exponent of $a$. We will use this improvement in Section 8 since Lemma 10 provides actually an expected sufficient condition. The next lemma is a global version of the previous one.

Lemma 12 Let $\widetilde{C}_{J}$ be a connected component of $C_{J}$ in $L^{2}(0, T)$. We denote by $l$ the value of $J(\varepsilon)$ for all $\varepsilon \in \widetilde{C}_{J}$ and we set $\widetilde{J}(\varepsilon)=l-J(\varepsilon)$. There exist $\widetilde{\sigma}>0, \widetilde{\kappa}>0$ and $\left.\left.\widetilde{\theta} \in\right] 0, \frac{1}{2}\right]$ such that:

$$
\forall \varepsilon \in L^{2}(0, T), d_{2}\left(\varepsilon, \widetilde{C}_{J}\right)<\widetilde{\sigma}, \quad\|\nabla J(\varepsilon)\|_{L^{2}(0, T)} \geq \widetilde{\kappa}|\widetilde{J}(\varepsilon)|^{1-\widetilde{\theta}},
$$

where $d_{2}$ is the distance associated to the $L^{2}(0, T)$-norm. 
Proof: Lemma 11 ensures that for each point $a$ in $\widetilde{C}_{J}$ there exist three real numbers $\sigma_{a}$, $\theta_{a}$ and $\kappa_{a}$ such that:

$$
\forall \varepsilon \in \mathbb{R}^{N},\|\varepsilon-a\|_{L^{2}(0, T)}<\sigma_{a} \quad\|\nabla J(\varepsilon)\|_{L^{2}(0, T)} \geq \kappa_{a}|\widetilde{J}(\varepsilon)|^{1-\theta_{a}} .
$$

The compactness of $\widetilde{C}_{J}$, guaranteed by Lemma 6 , allows us to extract from $\left\{B\left(a, \frac{\sigma_{a}}{2}\right), a \in \widetilde{C}_{J}\right\}$ a finite family $A=\left\{B\left(a_{i}, \frac{\sigma_{a_{i}}}{2}\right)\right\}_{i \in F}$, where $F$ is a finite set of indexes, such that $\widetilde{C}_{J} \subset A$.

We then define $\widetilde{\sigma}, \widetilde{\kappa}$ and $\widetilde{\theta} \in] 0,1 / 2]$ as the respective lower bounds of $\left\{\frac{\sigma_{a_{i}}}{2}\right\}_{i \in F},\left\{\kappa_{a_{i}}\right\}_{i \in F}$ and $\left\{\theta_{a_{i}}\right\}_{i \in F}$ and the result follows.

\section{Optimizing sequence}

We have now gathered all the necessary results to present and analyze the optimizing sequence.

\subsection{Definition of the sequence}

Following the approach sketched in the introduction, Y. Maday and G. Turinici have defined an optimizing sequence $\left(\varepsilon^{k}\right)_{k \in \mathbb{N}}$ for the cost functional $J$ as follows [20] :

Consider $(\delta, \eta) \in] 0,2[\times] 0,2\left[, \varepsilon^{0} \in L^{\infty}(0, T), \widetilde{\varepsilon}^{0} \in L^{\infty}(0, T), \psi^{0}\right.$ and $\chi^{0}$ the corresponding solutions of (15) and (16) according to Lemma 1. The functions $\varepsilon^{k}$ and $\widetilde{\varepsilon}^{k}$ are computed by solving iteratively:

$$
\begin{aligned}
& \left\{\begin{array}{c}
i \partial_{t} \psi^{k}(x, t)=\left(H(x)-\varepsilon^{k}(t) \mu(x)\right) \psi^{k}(x, t) \\
\psi^{k}(x, 0)=\psi_{0}(x)
\end{array}\right. \\
& \varepsilon^{k}(t)=(1-\delta) \tilde{\varepsilon}^{k-1}(t)-\frac{\delta}{\alpha} \operatorname{Im}\left\langle\chi^{k-1}(t)|\mu| \psi^{k}(t)\right\rangle \\
& \left\{\begin{array}{l}
i \partial_{t} \chi^{k}(x, t)=\left(H(x)-\tilde{\varepsilon}^{k}(t) \mu(x)\right) \chi^{k}(x, t) \\
\chi^{k}(x, T)=O \psi^{k}(x, T)
\end{array}\right. \\
& \tilde{\varepsilon}^{k}(t)=(1-\eta) \varepsilon^{k}(t)-\frac{\eta}{\alpha} \operatorname{Im}\left\langle\chi^{k}(t)|\mu| \psi^{k}(t)\right\rangle .
\end{aligned}
$$

Existence and uniqueness of solutions $\psi^{k}$ and $\chi^{k}$ of the above equations result from an easy adaptation of Lemma 3, as for the proof of $\varepsilon^{k}, \tilde{\varepsilon}^{k} \in L^{2}(0, T)$ for all $k \in \mathbb{N}$.

Remark 2 : Note that this choice of optimizing sequence is not canonical. There exists other ways to guarantee that the condition (1) is fulfilled (see, e.g. [30]). However, this formulation includes many monotonic algorithms, e.g. the one by Krotov (presented in [29]) or by W. Zhu and H. Rabitz [34] which are often used in the numerical simulations.

\subsection{Properties of the sequence}

We present here two results about the sequence $\left(\varepsilon^{k}\right)_{k \in \mathbb{N}}$. The proofs can be found in [19, 20]. These results state that $\left(\varepsilon^{k}\right)_{k \in \mathbb{N}}$ defined by $(31)-(34)$ is bounded in $L^{\infty}(0, T)$ and that the corresponding sequence $\left(J\left(\varepsilon^{k}\right)\right)_{k \in \mathbb{N}}$ increases monotonically.

Lemma 13 Given an initial field $\varepsilon^{0} \in L^{\infty}(0, T)$, let us define $M$ by:

$$
M=\max \left(\left\|\varepsilon^{0}\right\|_{L^{\infty}(0, T)}, \max \left(1, \frac{\delta}{2-\delta}, \frac{\eta}{2-\eta}\right) \frac{\|O\|_{*}\|\mu\|_{L^{\infty}}}{\alpha}\right) .
$$


The sequences $\left(\varepsilon^{k}\right)_{k \in \mathbb{N}}$ and $\left(\widetilde{\varepsilon}^{k}\right)_{k \in \mathbb{N}}$ satisfy:

$$
\forall k \in \mathbb{N},\left\|\varepsilon^{k}\right\|_{L^{\infty}(0, T)} \leq M,\left\|\widetilde{\varepsilon}^{k}\right\|_{L^{\infty}(0, T)} \leq M .
$$

Lemma 14 The sequence $\left(\varepsilon^{k}\right)_{k \in \mathbb{N}}$ defined by (31)--(34) ensures the monotonic convergence of the cost functional $J$ in the sense that:

$$
\begin{aligned}
J\left(\varepsilon^{k+1}\right)-J\left(\varepsilon^{k}\right)= & \left\langle\psi^{k+1}(T)-\psi^{k}(T)|O| \psi^{k+1}(T)-\psi^{k}(T)\right\rangle \\
& +\left(\frac{2}{\eta}-1\right)\left\|\varepsilon^{k+1}-\widetilde{\varepsilon}^{k}\right\|_{L^{2}(0, T)}^{2}+\left(\frac{2}{\delta}-1\right)\left\|\widetilde{\varepsilon}^{k}-\varepsilon^{k}\right\|_{L^{2}(0, T)}^{2},
\end{aligned}
$$

and there exists $l_{\varepsilon^{0}}$ such that $\lim _{k \rightarrow+\infty} J\left(\varepsilon^{k}\right)=l_{\varepsilon^{0}}$.

In order to study the convergence of $\left(\varepsilon^{k}\right)_{k \in \mathbb{N}}$, we will need to estimate the gradient of $J$ at each point $\varepsilon^{k}$. Such an estimate is obtained in the next lemma.

Lemma 15 There exists $\lambda>0$, depending on $\mu, O, \alpha, \delta, \eta$ and $T$, such that:

$$
\left\|\nabla J\left(\varepsilon^{k}\right)\right\|_{L^{1}(0, T)} \leq \lambda\left(\left\|\varepsilon^{k}-\widetilde{\varepsilon}^{k-1}\right\|_{L^{2}(0, T)}+\left\|\widetilde{\varepsilon}^{k-1}-\varepsilon^{k-1}\right\|_{L^{2}(0, T)}\right) .
$$

Proof: Thanks to (20), we have:

$$
\begin{aligned}
\nabla J\left(\varepsilon^{k}\right)(t) & =-2\left(\alpha \varepsilon^{k}(t)+\operatorname{Im}\left\langle\chi^{k-1}(t)|\mu| \psi^{k}(t)\right\rangle+\operatorname{Im}\left\langle\chi^{\varepsilon^{k}}(t)-\chi^{k-1}(t)|\mu| \psi^{k}(t)\right\rangle\right) \\
& =-2\left(\alpha\left(1-\frac{1}{\delta}\right)\left(\varepsilon^{k}(t)-\widetilde{\varepsilon}^{k-1}(t)\right)+\operatorname{Im}\left\langle\chi^{\varepsilon^{k}}(t)-\chi^{k-1}(t)|\mu| \psi^{k}(t)\right\rangle\right)
\end{aligned}
$$

where $\chi^{\varepsilon^{k}}$ is the solution of (16) with $\psi=\psi^{k}$ and $\varepsilon=\varepsilon^{k}$.

Next, $\chi^{\varepsilon^{k}}-\chi^{k-1}$ is the solution of equation (17) corresponding to $\varepsilon=\varepsilon^{k}, \varepsilon^{\prime}=\widetilde{\varepsilon}^{k-1}-\varepsilon^{k}$ and $\chi=\chi^{k-1}$. The associated estimate (18) then gives:

$$
\begin{aligned}
\left\|\chi^{\varepsilon^{k}}(t)-\chi^{k-1}(t)\right\|_{L^{2}} & \leq 2\|\mu\|_{L^{\infty}}\left\|\varepsilon^{k}-\widetilde{\varepsilon}^{k-1}\right\|_{L^{1}(0, T)}\|O\|_{*}+\left\|O\left(\psi^{k}(T)-\psi^{k-1}(T)\right)\right\|_{L^{2}} \\
& \leq 4\|\mu\|_{L^{\infty}}\|O\|_{*}\left(\left\|\varepsilon^{k}-\widetilde{\varepsilon}^{k-1}\right\|_{L^{1}(0, T)}+\left\|\widetilde{\varepsilon}^{k-1}-\varepsilon^{k-1}\right\|_{L^{1}(0, T)}\right) .
\end{aligned}
$$

Combining (37) and (38), we obtain (36) with $\lambda=2 \sqrt{T}\left(4 T\|O\|_{*}\|\mu\|_{L^{\infty}}^{2}+\alpha\left(1-\frac{1}{\delta}\right)\right)$.

\subsection{Limit points of the sequence}

We now present some result about the limit points of $\left(\varepsilon^{k}\right)_{k \in \mathbb{N}}$. These results give first hints about the relationship between these limit points and the set $C_{J}$ of the critical points of the cost functional $J$. Thus, we obtain a first case of convergence.

Lemma 16 Let $\left(\varepsilon^{k_{n}}\right)_{n \in \mathbb{N}}$ be a weakly convergent sub-sequence of $\left(\varepsilon^{k}\right)_{k \in \mathbb{N}}$ in $L^{2}(0, T)$. Then $\left(\varepsilon^{k_{n}}\right)_{n \in \mathbb{N}}$ converges in $L^{\infty}(0, T)$ towards a critical point of the cost functional $J$.

Proof: Let $\left(\varepsilon^{k_{n}}\right)_{n \in \mathbb{N}}$ be a weakly convergent sub-sequence of $\left(\varepsilon^{k}\right)_{k \in \mathbb{N}}$ in $L^{2}(0, T)$ and let us consider $\ell \in \mathbb{N}$. Equation (35) ensures that $\left(\varepsilon^{k_{n}+\ell}\right)_{n \in \mathbb{N}}$ also converges weakly (and has the same limit as $\left.\left(\varepsilon^{k_{n}}\right)_{n \in \mathbb{N}}\right)$. Thanks to Lemma 5 , the sequences $\left(\chi^{k_{n}+\ell}\right)_{n \in \mathbb{N}}$ and $\left(\psi^{k_{n}+\ell}\right)_{n \in \mathbb{N}}$ converge strongly in the space $C\left([0, T] ; L^{2}\right)$. Thus, we obtain by bilinearity the strong convergence of both sequences $\left(\left\langle\chi^{k_{n}}|\mu| \psi^{k_{n}}\right\rangle\right)_{n \in \mathbb{N}}$ and $\left(\left\langle\chi^{k_{n}}|\mu| \psi^{k_{n}+1}\right\rangle\right)_{n \in \mathbb{N}}$ in $L^{\infty}(0, T)$. 
According to $(32)$ and $(34),\left(\varepsilon^{k_{n}}\right)_{n \in \mathbb{N}}$ also reads:

$$
\varepsilon^{k_{n}+1}=\underbrace{(1-\delta)(1-\eta)}_{\nu} \varepsilon^{k_{n}}+u_{k_{n}}
$$

where $|\nu|<1$ and where $u_{k_{n}}(t)=-\frac{(1-\delta) \eta}{\alpha} \operatorname{Im}\left\langle\chi^{k_{n}}(t)|\mu| \psi^{k_{n}}(t)\right\rangle-\frac{\delta}{\alpha} \operatorname{Im}\left\langle\chi^{k_{n}}(t)|\mu| \psi^{k_{n}+1}(t)\right\rangle$ strongly converges in $L^{\infty}(0, T)$. Note again that given $\ell \in \mathbb{N},\left(u_{k_{n}+\ell}\right)_{n \in \mathbb{N}}$ also converges in $L^{\infty}(0, T)$ (towards the same limit). For all $k \in \mathbb{N}$, the absolute value of $u_{k}(t)$ can be estimated by:

$$
\left|u_{k}(t)\right| \leq m=\frac{4\|\mu\|_{L^{\infty}}\|O\|_{*}}{\alpha} .
$$

Let us prove that $\left(\varepsilon^{k_{n}}\right)_{n \in \mathbb{N}}$ is Cauchy in $L^{\infty}(0, T)$. Consider $e>0$. There exists $n_{1}>0$ be such that

$$
2 m \sum_{j_{1}}^{\infty}|\nu|^{j} \leq \frac{e}{4}
$$

Since the sequence $\left(u_{k_{n}-\ell}\right)_{n \in \mathbb{N}}$ is Cauchy for all $\ell$ with $0 \leq \ell \leq n_{1}$, we have:

$$
\exists n_{2}>0 / \forall s>n_{2}, \forall q \geq 0,\left\|u_{k_{s+q}-\ell}-u_{k_{s}-\ell}\right\|_{L^{\infty}(0, T)} \leq \frac{e}{4 n_{1}} .
$$

Let $n$ be an integer fulfilling the conditions:

$$
\forall p \geq 0,\left|\nu^{k_{n+p}}-\nu^{k_{n}}\right| \leq \frac{e}{4\left\|\varepsilon^{0}\right\|_{L^{\infty}(0, T)}}, \quad k_{n}>n_{1}, \quad n>n_{2} .
$$

Let $p$ be a positive integer. Since we have, for all $n \in \mathbb{N}^{*}, \varepsilon^{k_{n}}=\nu^{k_{n}} \varepsilon^{0}+\sum_{j=0}^{k_{n}-1} \nu^{j} u_{k_{n}-j-1}$ we obtain

$$
\begin{aligned}
\varepsilon^{k_{n+p}}-\varepsilon^{k_{n}}= & \left(\nu^{k_{n+p}}-\nu^{k_{n}}\right) \varepsilon^{0}+\sum_{j=k_{n}}^{k_{n+p}-1} \nu^{j} u_{k_{n+p}-j-1} \\
& +\sum_{j_{1}}^{k_{n}-1} \nu^{j}\left(u_{k_{n+p}-j-1}-u_{k_{n}-j-1}\right)+\sum_{j=0}^{n_{1}-1} \nu^{j}\left(u_{k_{n+p}-j-1}-u_{k_{n}-j-1}\right) .
\end{aligned}
$$

Thank to (39) and the two first conditions of (41):

$$
\begin{gathered}
\left\|\left(\nu^{k_{n+p}}-\nu^{k_{n}}\right) \varepsilon^{0}\right\|_{L^{\infty}(0, T)} \leq \frac{e}{4}, \\
\left\|\sum_{j=k_{n}}^{k_{n+p}-1} \nu^{j} u_{k_{n+p}-j-1}\right\|_{L^{\infty}(0, T)} \leq \sum_{j=k_{n}}^{\infty}\left\|\nu^{j} u_{k_{n+p}-j-1}\right\|_{L^{\infty}(0, T)} \leq m \sum_{j=k_{n}}^{\infty}|\nu|^{j} \leq \frac{e}{4} .
\end{gathered}
$$

According to the condition (39), the third term of (42) can be estimated by:

$$
\left\|\sum_{j_{1}}^{k_{n}-1} \nu^{j}\left(u_{k_{n+p}-j-1}-u_{k_{n}-j-1}\right)\right\|_{L^{\infty}(0, T)} \leq 2 m \sum_{j_{1}}^{\infty}|\nu|^{j} \leq \frac{e}{4}
$$

Lastly, $|\nu|<1$, the third condition of (41) and the Cauchy property (40) allows us to estimate the last term of (42):

$$
\left\|\sum_{j=0}^{n_{1}-1} \nu^{j}\left(u_{k_{n+p}-j-1}-u_{k_{n}-j-1}\right)\right\|_{L^{\infty}(0, T)} \leq \sum_{j=0}^{n_{1}-1}\left\|u_{k_{n+p}-j-1}-u_{k_{n}-j-1}\right\|_{L^{\infty}(0, T)} \leq \frac{e}{4} .
$$


We have thus proved that for all $e>0$, if $n$ is large enough then, for every $p>0$,

$$
\left\|\varepsilon^{k_{n+p}}-\varepsilon^{k_{n}}\right\|_{L^{\infty}(0, T)} \leq e,
$$

which proves that $\left(\varepsilon^{k_{n}}\right)_{n \in \mathbb{N}}$ is Cauchy in $L^{\infty}(0, T)$.

We denote by $\varepsilon$ the limit of $\left(\varepsilon^{k_{n}}\right)_{n \in \mathbb{N}}$. Thanks to (35), $\left(\widetilde{\varepsilon}^{k_{n}}\right)_{n \in \mathbb{N}}$ also converges towards $\varepsilon$. Passing through the limit in $(31)-(34)$, we then deduce that $\varepsilon$ belongs to $C_{J}$, according to definition (21).

Let us denote by $C_{\varepsilon^{0}} \subset C_{J}$ the set of the limit points of $\left(\varepsilon^{k_{n}}\right)_{n \in \mathbb{N}}$. As stated in Remark 1 , for $\alpha>6 T\|\mu\|_{L^{\infty}}^{2}\|O\|_{*}, C_{J}$, and consequently $C_{\varepsilon^{0}}$, are reduced to one point. By means of Lemma 13 , the convergence of the sequence $\left(\varepsilon^{k}\right)_{k \in \mathbb{N}}$ is then guaranteed in this case.

Remark 3 : In addition, the uniqueness of the critical point implies that the limit in this case is necessarily an extremum of $J$.

In order to obtain the convergence for all $\alpha>0$, we need to study more precisely the asymptotic behavior of the sequence $\left(\varepsilon^{k}\right)_{k \in \mathbb{N}}$ in the neighborhood of $C_{\varepsilon^{0}}$. A standard argument of compactness applied to $C_{\varepsilon^{0}}$ enables us to obtain the following result.

Lemma 17 Let denote by $d_{\infty}$ the distance corresponding to the $L^{\infty}(0, T)$ norm. One has:

$$
d_{\infty}\left(\varepsilon^{k}, C_{\varepsilon^{0}}\right) \rightarrow 0 .
$$

Remark 4 : By means of the monotonicity property, we find that $J=l_{\varepsilon^{0}}$ on the set $C_{\varepsilon^{0}}$ (with $l_{\varepsilon^{0}}=\lim _{k \rightarrow+\infty} J\left(\varepsilon^{k}\right)$ ). It is then possible to apply Lemma 12 with $\widetilde{C}_{J}=C_{\varepsilon^{0}}$ since the

assumption that $\widetilde{C}_{J}$ is connected is only necessary to ensure that $J$ is constant on this set. It can however be proved that $C_{\varepsilon^{0}}$ is connected (see [25]).

\section{Convergence of the sequence}

It is now possible to prove the convergence of the sequence $\left(\varepsilon^{k}\right)_{k \in \mathbb{N}}$ by a Cauchy argument.

Theorem 2 Suppose that $\varepsilon^{0} \in L^{\infty}(0, T)$. The sequence $\left(\varepsilon^{k}\right)_{k \in \mathbb{N}}$ defined by $(31)-(34)$ is convergent in $L^{2}(0, T)$.

Proof: We still denote by $l_{\varepsilon^{0}}$ the value of $J$ on $C_{\varepsilon^{0}}$ and by $\widetilde{J}$ the shifted cost functional $J-l_{\varepsilon^{0}}$. Suppose first that $\forall k \in \mathbb{N}, \widetilde{J}\left(\varepsilon^{k}\right) \neq 0$. By (43), there exists $k_{0}$ such that (30) holds (with $\widetilde{C}_{J}=C_{\varepsilon^{0}}$ ) for all $\varepsilon^{k}$ with $k \geq k_{0}$. Consider an integer $k \geq k_{0}$. We have:

$$
\begin{aligned}
\left(\left(\widetilde{J}\left(\varepsilon^{k}\right)\right)^{\widetilde{\theta}}-\left(\widetilde{J}\left(\varepsilon^{k+1}\right)\right)^{\tilde{\theta}}\right) & \geq \frac{\widetilde{\theta}}{\left(\widetilde{J}\left(\varepsilon^{k+1}\right)\right)^{1-\widetilde{\theta}}}\left(J\left(\varepsilon^{k+1}\right)-J\left(\varepsilon^{k}\right)\right) \\
& \geq \frac{\widetilde{\kappa} \tilde{\theta}}{\left\|\nabla J\left(\varepsilon^{k+1}\right)\right\|_{L^{1}(0, T)}}\left(\left(\frac{2}{\delta}-1\right)\left\|\varepsilon^{k+1}-\tilde{\varepsilon}^{k}\right\|_{L^{2}(0, T)}^{2}\right. \\
& \left.+\left(\frac{2}{\eta}-1\right)\left\|\widetilde{\varepsilon}^{k}-\varepsilon^{k}\right\|_{L^{2}(0, T)}^{2}\right) \\
& \geq \frac{\widetilde{\kappa} \widetilde{\theta} a_{(\delta, \eta)}}{\lambda}\left(\left\|\varepsilon^{k+1}-\tilde{\varepsilon}^{k}\right\|_{L^{2}(0, T)}+\left\|\tilde{\varepsilon}^{k}-\varepsilon^{k}\right\|_{L^{2}(0, T)}\right) \\
& \geq \frac{\widetilde{\kappa} \tilde{\theta} a_{(\delta, \eta)}}{\lambda}\left\|\varepsilon^{k+1}-\varepsilon^{k}\right\|_{L^{2}(0, T)},
\end{aligned}
$$


where $a_{(\delta, \eta)}=\frac{1}{\max (\delta, \eta)}-\frac{1}{2}$. The inequality (44) comes from the concavity of $s \mapsto s^{\tilde{\theta}}$, whereas (45) is a consequence of (30) and (35). Inequality (46) follows from (36).

Since $\left(\left(\widetilde{J}\left(\varepsilon^{k}\right)\right)^{\widetilde{\theta}}\right)_{k \in \mathbb{N}}$ is a Cauchy sequence (as a monotonic sequence bounded by $\left(2\|O\|_{*}\right)^{\widetilde{\theta}}$ ), we obtain that $\left(\varepsilon^{k}\right)_{k \in \mathbb{N}}$ is also a Cauchy sequence.

If there exists $k_{1}$ such that $\widetilde{J}\left(\varepsilon^{k_{1}}\right)=0$, the monotonicity of the algorithm implies that

$$
J\left(\varepsilon^{k_{1}}\right)=J\left(\varepsilon^{k_{1}+1}\right)=J\left(\varepsilon^{k_{1}+2}\right)=\ldots
$$

and by (35) the sequence $\left(\varepsilon^{k}\right)_{k \in \mathbb{N}}$ is constant for $k \geq k_{1}$.

Remark 5 : Thanks to the definition of the sequence $\left(\varepsilon^{k}\right)_{k \in \mathbb{N}}$ and to the regularity of the solutions $\psi$ and $\chi$ of the appropriate Schrödinger equations (see lemmas 1 to 3), we can easily prove by induction that if $\varepsilon^{0} \in W^{1, \infty}(0, T)$, then for all $k \in \mathbb{N}, \varepsilon^{k} \in W^{1, \infty}(0, T)$.

\section{Rate of convergence}

The rate of convergence can be now evaluated by a second use of the Łojasiewicz inequality. The result is summarized in the next theorem.

Theorem 3 Let us denote by $\varepsilon^{\infty}$, the limit of $\left(\varepsilon^{k}\right)_{k \in \mathbb{N}}$ defined by $(31)-(32)$ and $\widetilde{\theta}, \widetilde{\kappa}$ the real numbers appearing in (30), where $C_{\varepsilon^{0}}=\left\{\varepsilon^{\infty}\right\}$.

If $\widetilde{\theta}<\frac{1}{2}$, then there exists $c>0$ such that $\left\|\varepsilon^{k}-\varepsilon^{\infty}\right\|_{L^{2}(0, T)} \leq c k^{-\frac{\tilde{\theta}}{1-2 \theta}}$.
If $\widetilde{\theta}=\frac{1}{2}$, then there exist $c^{\prime}$ and $\tau$ such that:

$$
\left\|\varepsilon^{k}-\varepsilon^{\infty}\right\|_{L^{2}(0, T)} \leq c^{\prime} e^{-\tau k} .
$$

Proof: As in the proof of Theorem 2, let be $k_{0}$, an integer such that

$$
\forall \ell \geq k_{0} \quad\left\|\nabla J\left(\varepsilon^{\ell}\right)\right\|_{L^{1}(0, T)} \geq \widetilde{\kappa}\left|\widetilde{J}\left(\varepsilon^{\ell}\right)\right|^{1-\tilde{\theta}} .
$$

Let us fix $k \geq k_{0}$ and introduce $\Delta^{k}$ defined by:

$$
\Delta^{k}=\sum_{\ell=k}^{\infty}\left\|\varepsilon^{\ell+1}-\tilde{\varepsilon}^{\ell}\right\|_{L^{2}(0, T)}+\left\|\tilde{\varepsilon}^{\ell}-\varepsilon^{\ell}\right\|_{L^{2}(0, T)} .
$$

With no loss of generality, we may assume that $\Delta^{k}>0$ for all $k \geq k_{0}$. Summing (46) between $k$ and $+\infty$, we obtain:

$$
\left(\widetilde{J}\left(\varepsilon^{k}\right)\right)^{\tilde{\theta}} \geq \frac{\widetilde{\kappa} \tilde{\theta} a_{(\delta, \eta)}}{\lambda} \Delta^{k} .
$$

This estimate, combined with (48), with $\ell=k$ yields:

$$
\left\|\nabla J\left(\varepsilon^{k}\right)\right\|_{L^{1}(0, T)} \geq \widetilde{\kappa}\left(\frac{\widetilde{\kappa} \tilde{\theta} a_{(\delta, \eta)}}{\lambda} \Delta^{k}\right)^{\frac{1-\tilde{\theta}}{\tilde{\theta}}} .
$$

From Lemma 15, we obtain:

$$
\lambda\left(\Delta^{k-1}-\Delta^{k}\right) \geq \widetilde{\kappa}\left(\frac{\widetilde{\kappa} \widetilde{\theta} a_{(\delta, \eta)}}{\lambda} \Delta^{k}\right)^{\frac{1-\tilde{\theta}}{\tilde{\theta}}},
$$


which may be written as follows:

$$
\frac{\Delta^{k-1}-\Delta^{k}}{\left(\Delta^{k}\right)^{\beta}} \geq v
$$

with $\beta=\frac{1-\tilde{\theta}}{\tilde{\theta}}$ and $v=\frac{\widetilde{\kappa}}{\lambda}\left(\frac{\tilde{\kappa} \widetilde{\theta} a_{(\delta, \eta)}}{\lambda}\right)^{\frac{1-\tilde{\theta}}{\theta}}$. Suppose now that $\widetilde{\theta}=\frac{1}{2}$, i.e., $\beta=1$. The equation (49) then becomes:

$$
(1+v)^{k_{0}} \Delta^{k_{0}}\left(\frac{1}{1+v}\right)^{k} \geq \Delta^{k}
$$

and (47) is proved with $c^{\prime}=(1+v)^{k_{0}} \Delta^{k_{0}}$ and $\tau=\ln (1+v)$.

Suppose now that $\widetilde{\theta}<\frac{1}{2}$. Let be $\left.r \in\right] 0,1[$, and suppose first that:

$$
\left(\Delta^{k}\right)^{\beta} \geq r\left(\Delta^{k-1}\right)^{\beta} .
$$

Since $1-\beta<0$, the function $s \mapsto s^{1-\beta}$ is concave and we have:

$$
\left(\Delta^{k}\right)^{1-\beta}-\left(\Delta^{k-1}\right)^{1-\beta} \geq(\beta-1) \frac{\Delta^{k-1}-\Delta^{k}}{\left(\Delta^{k-1}\right)^{\beta}} \geq(\beta-1) r \frac{\Delta^{k-1}-\Delta^{k}}{\left(\Delta^{k}\right)^{\beta}} \geq(\beta-1) r v .
$$

In the other case:

$$
\left(\Delta^{k}\right)^{1-\beta}-\left(\Delta^{k-1}\right)^{1-\beta} \geq\left(\Delta^{k}\right)^{1-\beta}-\left(r^{\frac{1}{\beta}} \Delta^{k}\right)^{1-\beta}=\left(1-r^{\frac{1-\beta}{\beta}}\right)\left(\Delta^{k}\right)^{1-\beta} \geq\left(1-r^{\frac{1-\beta}{\beta}}\right)\left(\Delta^{k_{0}}\right)^{1-\beta} .
$$

Thus, in any case, there exists $v^{\prime}>0$ independent of $k$, such that:

$$
\left(\Delta^{k}\right)^{1-\beta}-\left(\Delta^{k-1}\right)^{1-\beta} \geq v^{\prime} .
$$

Consider now $k^{\prime}>k$, the inequality (50) implies that for a small enough $c$, one have:

$$
\Delta^{k^{\prime}} \leq\left(v^{\prime}\left(k^{\prime}-k\right)+\left(\Delta^{k}\right)^{2-\frac{1}{\theta}}\right)^{-\frac{\tilde{\theta}}{1-2 \theta}} \leq c k^{\prime-\frac{\tilde{\theta}}{1-2 \theta}},
$$

and the result follows.

Remark 6 : Thanks to Lemma 10, we have thus obtained that if $\alpha>6 T\|\mu\|_{L^{\infty}}^{2}\|O\|_{*}$ the convergence of the sequence is at least linear.

Acknowledgment: This work has been initiated at the summer school and workshop "Partial Differential Equations, Optimal Design and Numerics", organized by G. Buttazzo and E. Zuazua and is partially supported by the A.C.I "Simulation Moléculaire" of the french MENRT and by the Deutsche Forschungsgemeinschaft, SFB 404, B8. J.S. acknowledges helpful discussions on that topic with J. Bolte (Laboratoire de Combinatoire et Optimisation, Université Pierre \& Marie Curie, Paris) and O. Kavian (Laboratoire de Mathématiques, Université de Versailles Saint-Quentin). L.B. thanks G. Turinici (CEREMADE, Université Paris-Dauphine).

\section{Appendix: Proof of Lemma 11}

Consider $\varepsilon \in L^{2}(0, T)$ and $J$ defined by (14). For reason of simplicity, we suppose that $J(\varepsilon)=0, \nabla J(\varepsilon)=0$.

Thanks to Lemma 9, the operator $H_{J}(\varepsilon)$ is a Fredholm operator. The Fredholm alternative states then that either $H_{J}(\varepsilon)$ is bijective or $\operatorname{Ker} H_{J}(\varepsilon)=\operatorname{span}\left(\varphi_{1}, \ldots, \varphi_{m}\right)$, with $m>0$. Let 
us denote by $\Pi$, the orthogonal projection on $\operatorname{KerH}_{J}(\varepsilon)$ (with $\Pi=0$ if $\operatorname{KerH}_{J}(\varepsilon)=0$ ). The operator $L=\Pi+H_{J}(\varepsilon)$ is then bijective on $L^{2}(0, T)$.

We are now in the position to apply the local inverse mapping theorem to $\mathcal{L}=\Pi+\nabla J$ (analytic version, see [33], Corollary 4.37, p.172), that asserts there exist $V$ and $V^{\prime}$ two neighborhoods of 0 in $L^{2}(0, T)$ and $K: V^{\prime} \rightarrow V$ an analytic mapping such that:

$$
\forall \varepsilon^{\prime} \in V, K\left(\mathcal{L}\left(\varepsilon^{\prime}\right)\right)=\varepsilon^{\prime}, \quad \forall \varepsilon^{\prime \prime} \in V^{\prime}, \mathcal{L}\left(K\left(\varepsilon^{\prime \prime}\right)\right)=\varepsilon^{\prime \prime} .
$$

Since $\mathcal{L}$ and $K$ are $C^{\infty}$, there exist $C$ and $C^{\prime}$ such that:

$$
\begin{aligned}
\forall \varepsilon_{1}, \varepsilon_{2} \in V, \quad\left\|\mathcal{L}\left(\varepsilon_{2}\right)-\mathcal{L}\left(\varepsilon_{1}\right)\right\|_{L^{2}(0, T)} & \leq C\left\|\varepsilon_{2}-\varepsilon_{1}\right\|_{L^{2}(0, T)} \\
\forall \varepsilon_{1}^{\prime}, \varepsilon_{2}^{\prime} \in V^{\prime}, \quad\left\|K\left(\varepsilon_{2}^{\prime}\right)-K\left(\varepsilon_{1}^{\prime}\right)\right\|_{L^{2}(0, T)} & \leq C^{\prime}\left\|\varepsilon_{2}^{\prime}-\varepsilon_{1}^{\prime}\right\|_{L^{2}(0, T)} .
\end{aligned}
$$

Consider now $\varepsilon^{\prime} \in V \cap V^{\prime}$. For $\zeta \in \mathbb{R}^{m}$ such that $\sum_{j=1}^{m} \zeta_{j} \varphi_{j} \in V$, let us define $\Gamma: \zeta \mapsto$ $J\left(K\left(\sum_{j=1}^{m} \zeta_{j} \varphi_{j}\right)\right)$, and $\xi \in \mathbb{R}^{m}$ such that $\Pi \varepsilon^{\prime}=\sum_{j=1}^{m} \xi_{j} \varphi_{j}$. Let us first estimate $\nabla \Gamma(\xi)$. Using $\Pi \varepsilon^{\prime} \in V^{\prime}$, we obtain:

$$
\begin{aligned}
|\nabla \Gamma(\xi)| & \leq C^{\prime \prime}\left\|\nabla J\left(K\left(\Pi \varepsilon^{\prime}\right)\right)\right\|_{L^{2}(0, T)}=C^{\prime \prime}\left\|\nabla J\left(\varepsilon^{\prime}\right)+\nabla J\left(K\left(\Pi \varepsilon^{\prime}\right)\right)-\nabla J\left(\varepsilon^{\prime}\right)\right\|_{L^{2}(0, T)} \\
& \leq C^{\prime \prime}\left(\left\|\nabla J\left(\varepsilon^{\prime}\right)\right\|_{L^{2}(0, T)}+C\left\|K\left(\Pi \varepsilon^{\prime}\right)-\varepsilon^{\prime}\right\|_{L^{2}(0, T)}\right) \\
& =C^{\prime \prime}\left(\left\|\nabla J\left(\varepsilon^{\prime}\right)\right\|_{L^{2}(0, T)}+C\left\|K\left(\Pi \varepsilon^{\prime}\right)-K\left(\Pi \varepsilon^{\prime}+\nabla J\left(\varepsilon^{\prime}\right)\right)\right\|_{L^{2}(0, T)}\right) \\
& \leq c\left\|\nabla J\left(\varepsilon^{\prime}\right)\right\|_{L^{2}(0, T)}
\end{aligned}
$$

where $c=C^{\prime \prime}\left(1+C C^{\prime}\right)$. On the other hand, one has:

$$
\begin{aligned}
\left|J\left(\varepsilon^{\prime}\right)-\Gamma(\xi)\right| & =\left|J\left(\varepsilon^{\prime}\right)-J\left(K\left(\Pi \varepsilon^{\prime}\right)\right)\right|=\left|\int_{0}^{1} \frac{d}{d s} J\left(\varepsilon^{\prime}+s\left(K\left(\Pi \varepsilon^{\prime}\right)-\varepsilon^{\prime}\right)\right) d s\right| \\
& =\left|\int_{0}^{1}\left(\nabla J\left(\varepsilon^{\prime}+s\left(K\left(\Pi \varepsilon^{\prime}\right)-\varepsilon^{\prime}\right)\right), K\left(\Pi \varepsilon^{\prime}\right)-\varepsilon^{\prime}\right) d s\right| \\
& \left.\leq \| K\left(\Pi \varepsilon^{\prime}\right)-\varepsilon^{\prime}\right)\left\|_{L^{2}(0, T)} \int_{0}^{1}\right\| \nabla J\left(\varepsilon^{\prime}\right)\left\|_{L^{2}(0, T)}+C s\right\| K\left(\Pi \varepsilon^{\prime}\right)-\varepsilon^{\prime} \|_{L^{2}(0, T)} d s \\
& \left.=\| K\left(\Pi \varepsilon^{\prime}\right)-\varepsilon^{\prime}\right) \|_{L^{2}(0, T)}\left(\left\|\nabla J\left(\varepsilon^{\prime}\right)\right\|_{L^{2}(0, T)}+\frac{C}{2}\left\|K\left(\Pi \varepsilon^{\prime}\right)-\varepsilon^{\prime}\right\|_{L^{2}(0, T)}\right) \\
& \leq c^{\prime}\left\|\nabla J\left(\varepsilon^{\prime}\right)\right\|_{L^{2}(0, T)}^{2},
\end{aligned}
$$

where $c^{\prime}=C^{\prime}\left(1+\frac{C C^{\prime}}{2}\right)$. By diminishing $V$, the Eojasiewicz inequality (29) applied to the analytic functional $\Gamma$ states that there exist $\theta \in] 0,1 / 2], \sigma>0$ such that:

$$
\begin{aligned}
|\nabla \Gamma(\xi)| & \geq|\Gamma(\xi)|^{1-\theta}=\left|J\left(\varepsilon^{\prime}\right)-\Gamma(\xi)-J\left(\varepsilon^{\prime}\right)\right|^{1-\theta} \\
& \geq \frac{1}{2}\left|J\left(\varepsilon^{\prime}\right)\right|^{1-\theta}-\frac{1}{2}\left|J\left(\varepsilon^{\prime}\right)-\Gamma(\xi)\right|^{1-\theta} .
\end{aligned}
$$

Combining (51) and (52), we obtain:

$$
c\left\|\nabla J\left(\varepsilon^{\prime}\right)\right\|_{L^{2}(0, T)} \geq \frac{1}{2}\left|J\left(\varepsilon^{\prime}\right)\right|^{1-\theta}-c^{\prime}\left\|\nabla J\left(\varepsilon^{\prime}\right)\right\|_{L^{2}(0, T)}^{2(1-\theta)},
$$

and the result follows.

\section{References}

[1] A. Assion, T. Baumert, M. Bergt, T. Brixner, B. Kiefer, V. Seyfried, M. Strehle, G. Gerber, Control of chemical reactions by feedback-optimized phase-shaped femtosecond laser pulses, Science, 282 (1998) 919-922. 
[2] J. M. Ball, J. E. Marsden, M. Slemrod, Controlability for distributed bilinear systems, SIAM J. Cont. Opt., 20 (1982) 575-597.

[3] L. Baudouin, O. Kavian, J.-P. Puel, Regularity for a Schrödinger equation with singular potentials and application to bilinear optimal control, J. Diff. Eq., 216 (2005) 188-222.

[4] L. Baudouin, A bilinear optimal control problem applied to a time dependent Hartree-Fock equation coupled with classical nuclear dynamics, Portugaliae Mathematica (N.S.), 63 (1) (2006) 293-325.

[5] L. Baudouin, Existence and regularity of the solution of a time dependent Hartree-Fock equation coupled with a classical nuclear dynamics, Revista Matematica Complutense, 18 (2) (2005) $285-314$.

[6] K. Beauchard, Local controllability of a 1D Schrödinger equation, J. Math. Pures Appl., 84 (7) (2005) 851-956.

[7] T. Brixner, N. H. Damrauer, P. Niklaus, G. Gerber, Photoselective adaptive femtosecond quantum control in the liquid phase, Nature, 414 (2001) 57-60.

[8] E. Brown, H. Rabitz, Some mathematical and algorithmic challenges in the control of quantum dynamics phenomena, J. Math. Chem., 31 (2002) 17-63.

[9] E. Cancès, C. Le Bris, M. Pilot, Optimal bilinear control for a Schrödinger equation, C. R. Acad. Sci. Paris, 330 (Série 1) (2000) 567-571.

[10] E. Cancès, C. Le Bris, Y. Maday, G. Turinici, Mathematical Foundations of Molecular Modelling, Oxford Univ. Press, oxford, 2007.

[11] T. Cazenave, An introduction to nonlinear Schrödinger equation, third edition, Textos de Métodos Matemáticos 26, Rio de Janeiro, 1996.

[12] J. Kasparian, M. Rodriguez, G. Méjean, J. Yu, E. Salmon, H. Wille, R. Bourayou, S. Frey, Y.-B. Andr, A. Mysyrowicz, R. Sauerbrey, J.-P. Wolf, L. Woste, White-light filaments for atmospheric analysis, Science, 301 (2003) 61-64.

[13] A. Haraux, M.A. Jendoubi, O. Kavian, Rate of decay to equilibrium in some semilinear parabolic equations, J. Evol. Equ., 3 (2003) 463-484.

[14] K. Ito, K. Kunisch, Optimal bilinear control of an abstract Schrödinger equation, SIAM J. Cont. Opt. , to appear (2007).

[15] M. A. Jendoubi, A simple unified approach to some convergence theorems of L. Simon, J. Func. Anal., 153 (1998) 187-202.

[16] C. Le Bris, Computational Chemistry, Handbook of Numerical Analysis, Ph. G. Ciarlet ed., volume X, North-Holland, 2003.

[17] S. Łojasiewicz, Une propriété topologique des sous-ensembles analytiques réels, Colloques internationaux du CNRS. Les équations aux dérivées partielles, 117 (1963).

[18] S. Łojasiewicz, Sur la géométrie semi- et sous-analytique, Ann. Inst. Fourier, 43 (1993) 15751595.

[19] Y. Maday, J. Salomon, G. Turinici, Monotonic time-discretized schemes in quantum control, Num. Math., 103 (2) (2006) 323-338.

[20] Y. Maday, G. Turinici, New formulations of monotonically convergent quantum control algorithms, J. Chem. Phys, 118 (18) (2003).

[21] H. Rabitz, R. de Vivie-Riedle, M. Motzkus, K. Kompa, Whither the future of control- ling quantum phenomena?, Science, 288 (2000) 824-828.

[22] H. Rabitz, G. Turinici, E. Brown, Control of quantum dynamics: Concepts, procedures and future prospects, In Ph. G. Ciarlet, editor, Computational Chemistry, Special Volume (C. Le Bris Editor) of Handbook of Numerical Analysis, vol X, Elsevier Science B.V., 2003.

[23] M. Reed, B. Simon, Methods of Modern Mathematical Physics, II, Fourier analysis, selfadjointness, Academic Press, 1975.

[24] J. Salomon, Convergence of the time-discretized monotonic schemes, M2AN, 41 (1) (2007) 7793. 
[25] J. Salomon, Limit points of the monotonic schemes in quantum control, Proceedings of the 44th IEEE Conference on Decision and Control, Sevilla, 2005.

[26] J. Salomon, Contrôle en chimie quantique : conception et analyse de schémas d'optimisation, Thèse de l'Université Pierre et Marie Curie, 2005.

[27] S. Shi, A. Woody, H. Rabitz, Optimal control of selective vibrational excitation in harmonic linear chain molecules, J. Chem. Phys., 88 (1988) 6870-6883.

[28] L. Simon, Asymptotics for a class of non-linear evolution equations, with applications to geometric problems, Ann. of Math., 118 (1983) 525-571.

[29] D. Tannor, V. Kazakov, V. Orlov, Control of Photochemical Branching: Novel Procedures for Finding Optimal Pulses and Global Upper Bounds, in J. Broeckhove, L. Lathouwers (Eds), Time Dependent Quantum Molecular Dynamics, Plenum Press, New York, 1992, 347-360.

[30] G. Turinici, Monotonically Convergent Algorithms for Bounded Quantum Controls, Proceedings of the LHMNLC03 IFAC Conference, 2003 263-266.

[31] G. Vogt, G. Krampert, P. Niklaus, P. Nuernberger, G. Gerber, Optimal control of photoisomerization, Phys. Rev. Lett., 94: 68305 (2005).

[32] T. Weinacht, J. Ahn, P. Bucksbaum, Controlling the shape of a quantum wavefunction, Nature, 397 (1999) 233-235.

[33] E. Zeidler, Nonlinear functional analysis and its applications, tome 1, Springer-Verlag, Berlin/New York, 1985.

[34] W. Zhu, H. Rabitz, A rapid monotonically convergent algorithm for quantum optimal control over the expectation value of a definite operator, J. Chem. Phys., 109 (1998) 385-391. 